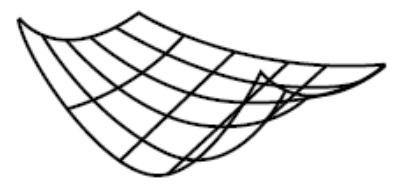

QUANTITATIVE FINANCE

RESEARCH CENTRE

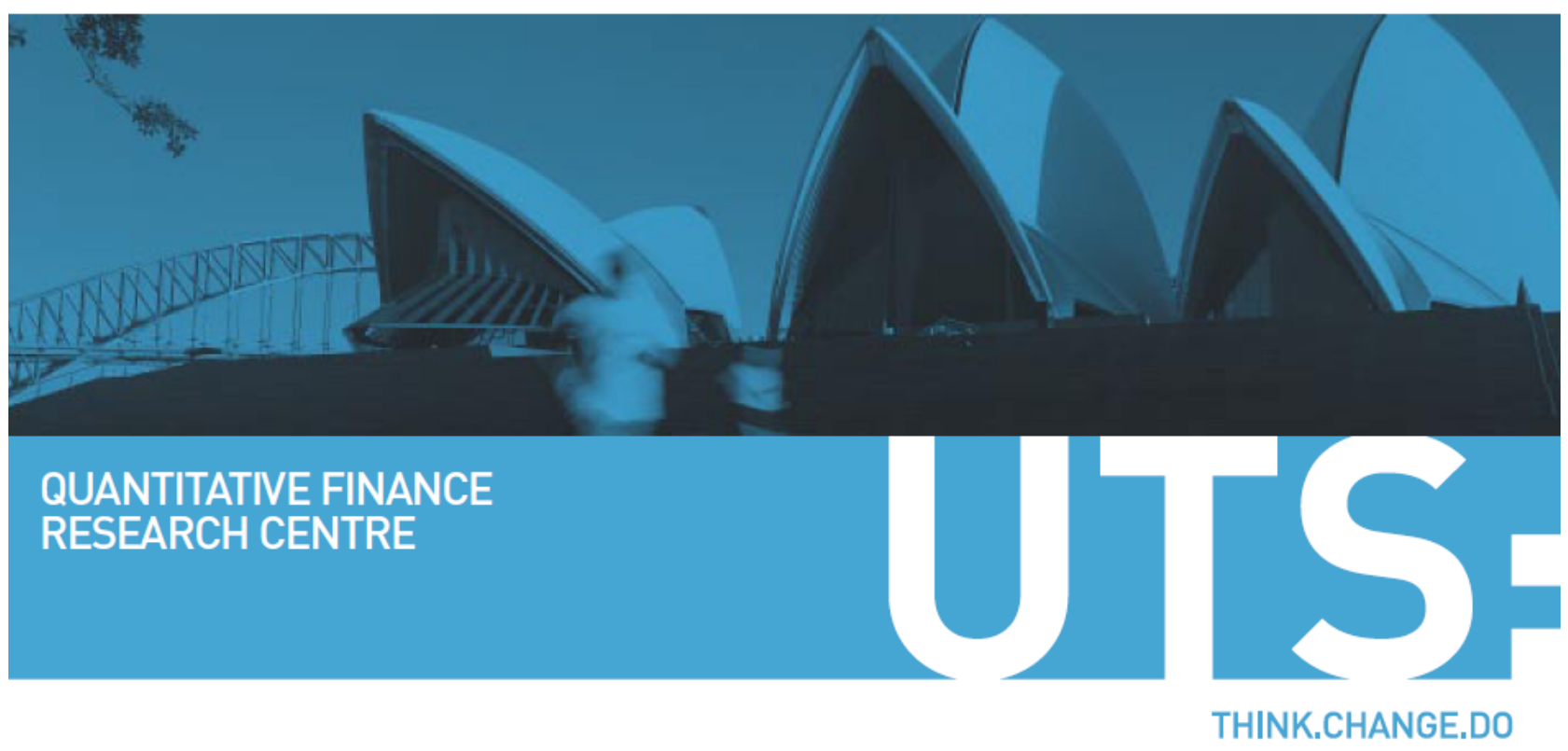

QUANTITATIVE FINANCE RESEARCH CENTRE

Credit Derivative Pricing with Stochastic Volatility Models

Carl Chiarella, Samuel Chege Maina and Christina Nikitopoulos Sklibosios 


\title{
Credit Derivative Pricing with Stochastic Volatility Models
}

\author{
Carl Chiarella, Samuel Chege Maina ${ }^{\dagger}$ Christina Nikitopoulos Sklibosios ${ }^{\ddagger}$ \\ Finance Discipline Group, UTS Business School \\ University of Technology, Sydney \\ P.O. Box 123, Broadway, NSW 2007, Australia
}

July 8, 2011

\begin{abstract}
This paper proposes a framework for pricing credit derivatives within the defaultable Markovian HJM framework featuring unspanned stochastic volatility. Motivated by empirical evidence, hump-shaped level dependent stochastic volatility specifications are proposed, such that the model admits finite dimensional Markovian structures. The model also accommodates a correlation structure between the stochastic volatility, default-free interest rates and credit spreads. Defaultfree and defaultable bonds are explicitly priced and an approach for pricing credit default swaps and swaptions is presented where the credit swap rates can be approximated by defaultable bond prices with varying maturities. A sensitivity analysis capturing the impact of the model parameters including correlations and stochastic volatility, on the credit swap rate and the value of the credit swaption is also presented.
\end{abstract}

Key Words: Stochastic volatility, Heath-Jarrow-Morton framework, defaultable bond prices, credit spreads, CDS rates.

*carl.chiarella@uts.edu.au

${ }^{\dagger}$ samuel.chege@uts.edu.au

${ }^{\ddagger}$ christina.nikitopoulos@uts.edu.au - Corresponding author. 


\section{Introduction}

Following the events of the recent financial crisis, both regulators and credit risk modellers have devoted special attention to better understanding credit risk markets and to further advance the models used to price and hedge the highly risky and elusive instruments traded in these markets.

Several key characteristics of interest rate markets which are closely related to credit risk markets have been identified in the literature. First, volatility in these markets is stochastic and includes elements that cannot be hedged by using only the underlying entities. In other words these markets feature unspanned stochastic volatility. CollinDufresne and Goldstein (2002), Casassus, Collin-Dufresne and Goldstein (2005) and Li and Zhao (2006) have demonstrated the existence of unspanned stochastic volatility factors in interest rate markets that drive the innovations of interest rate derivatives but do not affect the term structure. Second, there is empirical evidence that the volatility in these markets is hump-shaped. Reno and Uboldi (2005) argued that a model with hump-shaped term structure volatility could be an alternative to the unspanned volatility model of Collin-Dufresne and Goldstein (2002) and showed that hump-shaped volatility improves the model specifications, both in terms of yield errors and cap pricing performance. By using a default-free term structure model and a 7-year interest rate data set, Trolle and Schwartz (2009) found evidence of unspanned volatility components in interest rate markets and demonstrate that both hump-shaped term structure and unspanned stochastic volatility components are necessary to satisfactorily match the observed implied cap skews and implied volatilities. Third, innovations in interest rate volatility are correlated with innovations in interest rates, see for instance Ball and Torous (1999) and Trolle and Schwartz (2009). Fourth, interest rates are correlated with credit spreads. D'Souza, Amir-Atefi and Racheva-Jotova (2004) and Berndt, Ritchken and Sun (2010) demonstrate the importance of this correlation on pricing credit derivatives such as options on risky debt or credit insurance contracts.

The contributions of this paper are several. A generalised defaultable term structure model within the Heath, Jarrow and Morton (1992) (hereafter HJM) framework that accommodates unspanned stochastic volatility is presented. More specifically, the proposed model has the following properties. By construction, the model is consistent with the currently observed yield curve and credit spread curve. The model features a default-free term structure that is driven by $n$ factors and a defaultable term structure that is driven by $2 n$ factors. Additionally the stochastic volatility is specified by $3 n$ factors from which $n$ factors affect only (interest rate and credit) derivative prices. 
The connection between the two markets (default-free and defaultable) is established through the credit spread, as in Schönbucher (1998), which allows us to accommodate a correlation structure between interest rates, credit spreads and stochastic volatility.

However, it is well-known that these models are Markovian in the entire yield curve and credit spread curve thus requiring an infinite number of state variables. Consequently, a generalised volatility specification for the default-free and the defaultable term structure is proposed that leads to finite dimensional realisation of the state space, see for instance Chiarella and Kwon (2000a), Chiarella and Kwon (2000b), Björk, Landén and Svensson (2004) and Chiarella, Nikitopoulos-Sklibosios and Schlögl (2007). The proposed volatility structure, which depends on a Markovian volatility process, allows for level dependency and hump-shaped shocks. In this regard, our model can be considered as an extension of the Berndt et al. (2010) model to accommodate unspanned stochastic volatility. In line with the empirical evidence provided by Chan, Karolyi, Longstaff and Sanders (1992), Amin and Morton (1995) and Mercurio and Moraleda (2000), the volatility structure depends on the level of the short rates and the shortterm credit spreads. Under these volatility specifications, the model offers tractability and flexibility as it allows (default-free and defaultable) bond prices to be expressed as exponentially affine functions of state variables which are jointly Markovian. Although the model gives rise to a large (finite) number of state variables, their Markovian structure guarantees that the computational cost remains low.

The paper also considers the pricing of credit derivatives under the proposed model. We derive pricing formulas for single-name credit default swap rates (hereafter CDS rates) and swaptions. Based on approximations proposed by Brigo and Morini (2005), CDS rates are expressed in terms of defaultable bond prices with varying maturities, both in the absence and the presence of counterparty risk. Motivated by Rutkowski and Armstrong (2009), swaptions are also priced by using a Black's-type formula for options where the underlying is the CDS spread.

The final contribution of this paper is that an extensive sensitivity analysis is presented. Firstly, the impact of the full correlation structure and the volatility specifications on the distribution of the defaultable bond prices is analysed. The impact of the correlation structure and the stochastic volatility specifications on CDS rates and swaption prices is also studied. The results indicate that the correlation between the interest rate and credit spread does not have a significant impact on the CDS rate and consequently the swaption prices while the effect of the parameters of the stochastic volatility process is more profound. 
The paper is structured as follows. Section 2 presents a multi-dimensional defaultable term structure model with unspanned stochastic volatility. Section 3 proposes a hump-shaped level dependent volatility structure for the default-free and the defaultable forward rates that allow this model to admit finite dimensional affine realisations and to produce exponentially affine defaultable bond prices. Section 4 considers the pricing of credit default swaps and credit default swaptions. A sensitivity analysis of the impact of different parameter values on the credit default swaps and the credit default swaptions is carried out in Section 5. Section 6 concludes.

\section{A General Defaultable Term Structure Model}

We consider the filtered probability space $\left(\Omega, \mathcal{F},\left(\mathcal{F}_{t}\right)_{0 \leq t \leq T}, \mathbb{P}\right)$ where the filtration $\mathcal{F}_{t}=\mathcal{F}_{t}^{W} \vee \mathcal{F}_{t}^{\tau}, t \geq 0$, contains default-free information plus explicit monitoring of default up to time $t$ and satisfies the usual conditions. Here, $\mathbb{P}$ is the real world probability measure. More specifically, the subfiltration $\mathcal{F}_{t}^{W}$ represents all the default-free background information and is the $\sigma$-algebra generated by the standard $\mathbb{P}$-Wiener process $W(t)$,

$$
\left(\mathcal{F}_{t}^{W}\right)_{t \geq 0}=\{\sigma(W(s): 0 \leq s \leq t)\}_{t \geq 0}
$$

The default time $\tau$ is modelled as a stopping time defined by the first jump time of a Cox (doubly stochastic Poisson) process $N(t)=\sum_{i=1}^{\infty} \mathbb{1}_{\left\{\tau_{i} \leq t\right\}}$ with intensity $h(t)$, that is,

$$
\tau=\inf \left\{t \in \mathbb{R}^{+} \mid N(t)>0\right\} .
$$

In addition, $\mathcal{F}_{t}^{\tau}$ is the subfiltration generated by the counting indicator function $\mathbb{1}_{\{\tau \leq t\}}$,

$$
\left(\mathcal{F}_{t}^{\tau}\right)_{t \geq 0}=\left\{\sigma\left(\mathbb{1}_{\tau \leq s}: 0 \leq s \leq t\right)\right\}_{t \geq 0}
$$

The price at time $t$ of the default-free zero coupon bond with maturity $T>t$ is denoted as $P(t, T, \mathbb{V})$, where $\mathbb{V} \in \Omega$ represents the dependence on the stochastic volatility process at time $t$.

Definition 2.1 1. The instantaneous default-free forward rate of interest prevailing at time $t$ for instantaneous borrowing at the later time $T$, is defined as

$$
f(t, T, \mathbb{V})=-\frac{\partial}{\partial T} \ln P(t, T, \mathbb{V}), \quad \text { for all } t \in[0, T]
$$


2. The instantaneous default-free short rate is defined as the instantaneously maturing forward rate, so that

$$
r(t, \mathbb{V})=f(t, t, \mathbb{V})
$$

From definition (2.3), the default-free zero coupon bond price is expressed as

$$
P(t, T, \mathbb{V})=\exp \left(-\int_{t}^{T} f(t, s, \mathbb{V}) d s\right)
$$

We introduce next the defaultable term structure. We denote as $P^{d}(t, T, \mathbb{V})$ the price at time $t$ of the defaultable zero coupon bond with maturity $T>t$.

Definition 2.2 1. The instantaneous defaultable forward rate at time $t$ for instantaneous borrowing at $T$ is defined as

$$
f^{d}(t, T, \mathbb{V})=-\frac{\partial}{\partial T} \ln P^{d}(t, T, \mathbb{V}), \quad \text { for all } t \in[0, T]
$$

2. The instantaneous defaultable short rate is defined as

$$
r^{d}(t, \mathbb{V})=f^{d}(t, t, \mathbb{V})
$$

3. The continuously compounded instantaneous forward credit spread is defined as

$$
\lambda(t, T, \mathbb{V})=f^{d}(t, T, \mathbb{V})-f(t, T, \mathbb{V}),
$$

and the instantaneous short-term credit spread is defined as

$$
c(t, \mathbb{V})=\lambda(t, t, \mathbb{V})=r^{d}(t, \mathbb{V})-r(t, \mathbb{V}) .
$$

The $t$-value of the defaultable zero-coupon bond with maturity $T$, assuming that it has not defaulted up to time $t$, is denoted as $\bar{P}^{d}(t, T, \mathbb{V})$ (the so-called 'pseudo' bond), and is defined as

$$
\bar{P}^{d}(t, T, \mathbb{V})=\exp \left(-\int_{t}^{T} f^{d}(t, s, \mathbb{V}) d s\right)
$$

At default time $\tau$, the defaultable bond's face value is reduced by the loss $\operatorname{rat} 11 q(\tau) \in$ $[0,1]$, and its recovery value is given by

$$
\mathcal{R}(\tau):=1-q(\tau)
$$

\footnotetext{
${ }^{1}$ This is also referred to as the loss-given-default (LGD).
} 
Thus the value of the defaultable bond can be expressed as

$$
\begin{aligned}
P^{d}(t, T, \mathbb{V}) & =\mathcal{R}(t) \exp \left(-\int_{t}^{T} f^{d}(t, s, \mathbb{V}) d s\right) \\
& =\mathcal{R}(t) \bar{P}^{d}(t, T, \mathbb{V}) .
\end{aligned}
$$

To allow for various factors in the economy or the market, to drive the dynamics of the forward rate and credit spread, we propose the following multi-dimensional setup.

Assumption 2.1 The instantaneous default-free forward rate $f(t, T, \mathbb{V})$ and the instantaneous forward credit spread $\lambda(t, T, \mathbb{V})$ satisfy the stochastic integral equations

$$
\begin{aligned}
& f(t, T, \mathbb{V})=f\left(0, T, \mathbb{V}_{0}\right)+\int_{0}^{t} \alpha^{f}(u, T, \mathbb{V}) d u+\sum_{i=1}^{n} \int_{0}^{t} \sigma_{i}^{f}\left(u, T, V_{i}\right) d W_{i}^{f}(u), \\
& \lambda(t, T, \mathbb{V})=\lambda\left(0, T, \mathbb{V}_{0}\right)+\int_{0}^{t} \alpha^{\lambda}(u, T, \mathbb{V}) d u+\sum_{i=1}^{n} \int_{0}^{t} \sigma_{i}^{\lambda}\left(u, T, V_{i}\right) d W_{i}^{\lambda}(u),
\end{aligned}
$$

where the stochastic volatility vector process $\mathbb{V}=\left\{\left(V_{1}(t), \ldots, V_{n}(t)\right), t \in[0, T]\right\}$ satisfies the set of the stochastic differential equations

$$
d V_{i}(t)=\alpha_{i}^{\mathbb{V}}\left(t, V_{i}\right) d t+\sigma_{i}^{\mathbb{V}}\left(t, V_{i}\right) d W_{i}^{\mathbb{V}}(t), i=1,2, \ldots n
$$

and $\mathbb{V}_{0}$ is the initial value of the volatility process $\mathbb{V}$.

Note that the volatility functions $\sigma_{i}^{f}\left(t, T, V_{i}\right)$ and $\sigma_{i}^{\lambda}\left(t, T, V_{i}\right)$ depend only on the volatility process $V_{i}$ and that the drift $\alpha_{i}^{\mathbb{V}}\left(t, V_{i}\right)$ and the diffusion $\sigma_{i}^{\mathbb{V}}\left(t, V_{i}\right)$ of the each volatility process $V_{i}$ depends only on the $V_{i}$, for $i=1,2, \ldots, n$.

The stochastic integral equation for the defaultable forward rate is derived by using the definition (2.7) and the Assumption 2.1, where the dynamics of the defaultfree forward rate and forward credit spread are specified. Thus, by setting $f^{d}(0, T)=$ $f^{d}\left(0, T, \mathbb{V}_{0}\right)$, we obtain

$$
\begin{aligned}
f^{d}(t, T, \mathbb{V}) & =f^{d}(0, T)+\int_{0}^{t}\left(\alpha^{f}(u, T, \mathbb{V})+\alpha^{\lambda}(u, T, \mathbb{V})\right) d u \\
& +\sum_{i=1}^{n} \int_{0}^{t} \sigma_{i}^{f}\left(u, T, V_{i}\right) d W_{i}^{f}(u)+\sum_{i=1}^{n} \int_{0}^{t} \sigma_{i}^{\lambda}\left(u, T, V_{i}\right) d W_{i}^{\lambda}(u),
\end{aligned}
$$

where the initial defaultable forward curve is

$$
f^{d}(0, T)=f(0, T)+\lambda(0, T)
$$

For notational convenience, we set $f(0, t)=f\left(0, t, \mathbb{V}_{0}\right)$ and $\lambda(0, t)=\lambda\left(0, t, \mathbb{V}_{0}\right), t \in$ $[0, T]$. Furthermore, the specifications of Assumption 2.1 imply the following dynamics 
for the instantaneous default-free short rate $r(t, \mathbb{V}):=f(t, t, \mathbb{V})$ and the instantaneous short-term credit spread $c(t, \mathbb{V}):=\lambda(t, t, \mathbb{V})$

$$
\begin{aligned}
& r(t, \mathbb{V})=f(0, t)+\int_{0}^{t} \alpha^{f}(u, t, \mathbb{V}) d u+\sum_{i=1}^{n} \int_{0}^{t} \sigma_{i}^{f}\left(u, t, V_{i}\right) d W_{i}^{f}(u), \\
& c(t, \mathbb{V})=\lambda(0, t)+\int_{0}^{t} \alpha^{\lambda}(u, t, \mathbb{V}) d u+\sum_{i=1}^{n} \int_{0}^{t} \sigma_{i}^{\lambda}\left(u, t, V_{i}\right) d W_{i}^{\lambda}(u),
\end{aligned}
$$

respectively. For $T=t$, equation (2.15) yields the dynamics of the instantaneous defaultable short rate $r^{d}(t, \mathbb{V}):=f^{d}(t, t, \mathbb{V})=f(t, t, \mathbb{V})+\lambda(t, t, \mathbb{V})$ as

$$
\begin{aligned}
r^{d}(t, \mathbb{V}) & =f^{d}(0, t)+\int_{0}^{t}\left(\alpha^{f}(u, t, \mathbb{V})+\alpha^{\lambda}(u, t, \mathbb{V})\right) d u \\
& +\sum_{i=1}^{n} \int_{0}^{t} \sigma_{i}^{f}\left(u, t, V_{i}\right) d W_{i}^{f}(u)+\sum_{i=1}^{n} \int_{0}^{t} \sigma_{i}^{\lambda}\left(u, t, V_{i}\right) d W_{i}^{\lambda}(u) .
\end{aligned}
$$

In addition, we specify the correlation structure as follows

$$
\mathbb{E}\left[d W_{i}^{x} \cdot d W_{j}^{y}\right]=\left\{\begin{aligned}
\delta_{i j} \rho_{i}^{x y} d t & \text { if } x \neq y \\
\delta_{i j} d t & \text { if } x=y
\end{aligned}\right.
$$

where $\delta_{i j}=\left\{\begin{array}{ll}1 & \text { if } i=j, \\ 0 & \text { otherwise }\end{array}\right.$, for $x, y \in\{\mathbb{V}, \lambda, f\}, 1 \leq i, j \leq n$. and $\rho_{i}^{x y} \in[-1,1]$ for all $i$.

The correlated Wiener processes $W_{i}^{f}(t), W_{i}^{\lambda}(t)$ and $W_{i}^{\mathbb{V}}(t)$, for $i=1,2, \ldots, n$, can be expressed in terms of the independent Wiener processes $W_{i}(t)$, for $i=1,2, \ldots, 3 n$. For modelling convenience, we adopt the following decomposition (there are other possible decompositions)

$$
\begin{aligned}
& d W_{i}^{f}(t)=z_{i}^{f_{1}} d W_{i}(t) \\
& d W_{i}^{\lambda}(t)=z_{i}^{\lambda_{1}} d W_{i}(t)+z_{i}^{\lambda_{2}} d W_{n+i}(t) \\
& d W_{i}^{\mathbb{V}}(t)=z_{i}^{V_{1}} d W_{i}(t)+z_{i}^{V_{2}} d W_{n+i}(t)+z_{i}^{V_{3}} d W_{2 n+i}(t),
\end{aligned}
$$

where the correlation parameters, for $\left(\rho_{i}^{\lambda f}\right)^{2} \neq 1$, are set as

$$
\left\{\begin{array}{l}
z_{i}^{f_{1}}=1, \quad z_{i}^{\lambda_{1}}=\rho_{i}^{\lambda f}, \quad z_{i}^{\lambda_{2}}=\sqrt{1-\left(\rho_{i}^{\lambda f}\right)^{2}} \\
z_{i}^{V_{1}}=\rho_{i}^{V f}, \quad z_{i}^{V_{2}}=\frac{\rho_{i}^{V \lambda}-\rho_{i}^{\lambda f} \rho_{i}^{V f}}{\sqrt{1-\left(\rho_{i}^{\lambda f}\right)^{2}}}, \quad z_{i}^{V_{3}}=\sqrt{\frac{1-\left(\rho_{i}^{\lambda f}\right)^{2}-\left(\rho_{i}^{V f}\right)^{2}-\left(\rho_{i}^{V \lambda}\right)^{2}+2 \rho_{i}^{\lambda f} \rho_{i}^{V f} \rho_{i}^{V \lambda}}{1-\left(\rho_{i}^{\lambda f}\right)^{2}}} .
\end{array}\right.
$$

Then by using the decomposition (2.21), the stochastic integral equations (2.12) and (2.13), as well as, stochastic differential equation (2.14) are expressed in terms of inde- 
pendent Wiener processes as

$$
\begin{aligned}
f(t, T, V) & =f(0, T)+\int_{0}^{t} \alpha^{f}(u, T, \mathbb{V}) d u+\sum_{i=1}^{n} \int_{0}^{t} \tilde{\sigma}_{i}^{f}\left(u, T, V_{i}\right) d W_{i}(u), \\
\lambda(t, T, V) & =\lambda(0, T)+\int_{0}^{t} \alpha^{\lambda}(u, T, \mathbb{V}) d u+\sum_{i=1}^{2 n} \int_{0}^{t} \tilde{\sigma}_{i}^{\lambda}\left(u, T, V_{i}\right) d W_{i}(u), \\
d V_{i}(t) & =\alpha_{i}^{V}\left(t, V_{i}\right) d t+\tilde{\sigma}_{i 1}^{V}(t) d W_{i}(t)+\tilde{\sigma}_{i 2}^{V}(t) d W_{n+i}(t)+\tilde{\sigma}_{i 3}^{V}(t) d W_{2 n+i}(t),
\end{aligned}
$$

where the volatility functions $\tilde{\sigma}_{i}^{f}\left(t, T, V_{i}\right), \tilde{\sigma}_{i}^{\lambda}\left(t, T, V_{i}\right)$ and $\tilde{\sigma}_{i j}^{V}(t, T)$ are given by

$$
\tilde{\sigma}_{i}^{f}\left(t, T, V_{i}\right)= \begin{cases}z_{i}^{f_{1}} \sigma_{i}^{f}\left(t, T, V_{i}\right), & \text { for } i=1, \ldots, n \\ 0, & \text { otherwise }\end{cases}
$$

and

$$
\tilde{\sigma}_{i}^{\lambda}\left(t, T, V_{i}\right)= \begin{cases}z_{i}^{\lambda_{1}} \sigma_{i}^{\lambda}\left(t, T, V_{i}\right), & \text { for } i=1, \ldots, n \\ z_{i-n}^{\lambda_{2}} \sigma_{i-n}^{\lambda}\left(t, T, V_{i-n}\right), & \text { for } i=n+1, \ldots, 2 n\end{cases}
$$

while, for $j=1,2,3$ and $i=1, \ldots, n$

$$
\tilde{\sigma}_{i j}^{V}(t)=z_{i}^{V_{j}} \sigma_{i}^{\mathbb{V}}\left(t, V_{i}\right)
$$

Moreover, when the decomposition (2.21) is applied to (2.15) for the defaultable forward rate, we have that it follows the stochastic integral equation

$$
f^{d}(t, T, \mathbb{V})=f^{d}(0, T)+\int_{0}^{t} \alpha^{d}(u, T, \mathbb{V}) d u+\sum_{i=1}^{2 n} \int_{0}^{t} \tilde{\sigma}_{i}^{d}\left(u, T, V_{i}\right) d W_{i}(u),
$$

where

$$
\begin{aligned}
\alpha^{d}(u, T, \mathbb{V}) & =\alpha^{f}(u, T, \mathbb{V})+\alpha^{\lambda}(u, T, \mathbb{V}), \\
\tilde{\sigma}_{i}^{d}\left(t, T, V_{i}\right) & =\tilde{\sigma}_{i}^{f}\left(t, T, V_{i}\right)+\tilde{\sigma}_{i}^{\lambda}\left(t, T, V_{i}\right) .
\end{aligned}
$$

The system consisting of (2.23), (2.24) and (2.25) embeds stochastic volatility into a traditional defaultable HJM framework. The default-free forward rate curve is driven by $n$ sources of uncertainty, while the (apparently larger) defaultable forward rate curve is driven by $2 n$ sources of uncertainty. The volatility of these forward rate curves, in general, is driven by a total number of $3 n$ sources of uncertainty, subject to the correlation structure between the forward rates and their volatilities. Therefore, volatility sensitive instruments, such as interest rate derivatives and credit derivatives, are affected by $3 n$ factors. Indeed, the proposed framework can be considered as an adaption of the Heston (1993) stochastic volatility equity model, to a defaultable term structure model. 
Our extension of the HJM framework to a defaultable setting also incorporates unspanned stochastic volatility. A defaultable term structure model with $2 n$ factors is considered, where $n$ factors are associated with the default-free term structure and $3 n$ factors associated with their volatilities. Thus the proposed model, subject to the correlation structure, allows for up to $n$ unspanned stochastic volatility factors.

The absence of arbitrage implies that there exists an equivalent probability measure $\tilde{\mathbb{P}}$ where for every maturity $T$ there is a $3 n$-dimensional process

$$
\Phi_{n}(t)=\left\{\phi_{1}(t), \phi_{2}(t), \ldots, \phi_{3 n}(t), t \in[0, T]\right\}
$$

satisfying the integrability conditions

$$
\int_{0}^{t}\left\|\phi_{i}(s)\right\|^{2} d s<\infty, \quad \text { for } \quad i=1,2, \ldots, 3 n, \quad \int_{0}^{t}|\psi(s)| h(s) d s<\infty,
$$

such that

$$
d \tilde{W}_{i}(t)=d W_{i}(t)-\phi_{i}(t) d t, \quad \text { for } \quad i=1,2, \ldots, 3 n
$$

is a $\tilde{\mathbb{P}}$-Wiener process and the default indicator process $N(t)$ has a $\tilde{\mathbb{P}}$-intensity

$$
\tilde{h}(t)=\psi(t) h(t)
$$

Using Girsanov's theorem, and working along the lines of Heath et al. (1992) and Björk, Kabanov and Runggaldier (1997), we obtain the drift restriction for the HJM default-free forward rate and defaultable forward rate, respectively, as

$$
\begin{aligned}
& \alpha^{f}(t, T, \mathbb{V})=-\sum_{i=1}^{n} \tilde{\sigma}_{i}^{f}\left(t, T, V_{i}\right)\left(\phi_{i}(t)-\int_{t}^{T} \tilde{\sigma}_{i}^{f}\left(t, s, V_{i}\right) d s\right), \\
& \alpha^{d}(t, T, \mathbb{V})=-\sum_{i=1}^{2 n} \tilde{\sigma}_{i}^{d}\left(t, T, V_{i}\right)\left(\phi_{i}(t)-\int_{t}^{T} \tilde{\sigma}_{i}^{d}\left(t, s, V_{i}\right) d s\right) .
\end{aligned}
$$

Moreover, by using (2.36) together with (2.35) and (2.31), we derive the credit spread drift restriction which is expressed in terms of the volatilities of the default-free forward rate and the credit spread as (recall that $\tilde{\sigma}_{i}^{f}\left(t, T, V_{i}\right)=0$ for $\left.i=n+1, \ldots, 2 n\right)$

$$
\begin{aligned}
\alpha^{\lambda}(t, T, \mathbb{V}) & =-\sum_{i=1}^{2 n} \phi_{i}(t) \tilde{\sigma}_{i}^{\lambda}\left(t, T, V_{i}\right)+\sum_{i=1}^{2 n} \tilde{\sigma}_{i}^{\lambda}\left(t, T, V_{i}\right) \int_{t}^{T} \tilde{\sigma}_{i}^{\lambda}\left(t, s, V_{i}\right) d s \\
& +\sum_{i=1}^{n}\left(\tilde{\sigma}_{i}^{\lambda}\left(t, T, V_{i}\right) \int_{t}^{T} \tilde{\sigma}_{i}^{f}\left(t, s, V_{i}\right) d s+\tilde{\sigma}_{i}^{f}\left(t, T, V_{i}\right) \int_{t}^{T} \tilde{\sigma}_{i}^{\lambda}\left(t, s, V_{i}\right) d s\right) .
\end{aligned}
$$


By substituting the drift restrictions (2.35) and (2.37) into (2.23) and (2.24), respectively, we obtain the dynamics for the forward rate and forward credit spread processes under the risk neutral measure, namely

$$
\begin{aligned}
f(t, T, \mathbb{V})=f(0, T)+ & \sum_{i=1}^{n} \int_{0}^{t} \tilde{\sigma}_{i}^{f}\left(u, T, V_{i}\right) \int_{u}^{T} \tilde{\sigma}_{i}^{f}\left(u, s, V_{i}\right) d s d u+\sum_{i=1}^{n} \int_{0}^{t} \tilde{\sigma}_{i}^{f}\left(u, T, V_{i}\right) d \tilde{W}_{i}(u) \\
\lambda(t, T, \mathbb{V})=\lambda(0, T)+ & \sum_{i=1}^{2 n} \int_{0}^{t} \tilde{\sigma}_{i}^{\lambda}\left(u, T, V_{i}\right) \int_{u}^{T} \tilde{\sigma}_{i}^{\lambda}\left(u, s, V_{i}\right) d s d u+\sum_{i=1}^{n} \int_{0}^{t} \tilde{\sigma}_{i}^{\lambda}\left(u, T, V_{i}\right) \int_{u}^{T} \tilde{\sigma}_{i}^{f}\left(u, s, V_{i}\right) d s d u \\
& +\sum_{i=1}^{n} \int_{0}^{t} \tilde{\sigma}_{i}^{f}\left(u, T, V_{i}\right) \int_{u}^{T} \tilde{\sigma}_{i}^{\lambda}\left(u, s, V_{i}\right) d s d u+\sum_{i=1}^{t} \int_{0}^{t} \tilde{\sigma}_{i}^{\lambda}\left(u, t, V_{i}\right) d \tilde{W}_{i}(u) .
\end{aligned}
$$

Further, by substituting the drift restriction (2.36) into (2.29), the risk-neutral dynamics of the defaultable forward rate $f^{d}(t, T, \mathbb{V})$ are

$$
f^{d}(t, T, \mathbb{V})=f^{d}(0, T)+\sum_{i=1}^{2 n} \int_{0}^{t} \tilde{\sigma}_{i}^{d}\left(u, T, V_{i}\right)\left(\int_{u}^{T} \tilde{\sigma}_{i}^{d}\left(u, s, V_{i}\right) d s\right) d u+\sum_{i=1}^{2 n} \int_{0}^{t} \tilde{\sigma}_{i}^{d}\left(u, T, V_{i}\right) d \tilde{W}_{i}(u),
$$

where the risk neutral dynamics for the volatility process $\mathbb{V}=\left\{V_{1}, V_{2}, \ldots, V_{n}\right\}$ are

$$
\begin{array}{r}
d V_{i}(t)=\left[\alpha_{i}^{V}\left(t, V_{i}\right)+\phi_{i}(t) \tilde{\sigma}_{i 1}^{V}(t)+\phi_{n+i}(t) \tilde{\sigma}_{i 2}^{V}(t)+\phi_{2 n+i}(t) \tilde{\sigma}_{i 3}^{V}(t)\right] d t \\
+\tilde{\sigma}_{i 1}^{V}(t) d \tilde{W}_{i}(t)+\tilde{\sigma}_{i 2}^{V}(t) d \tilde{W}_{n+i}(t)+\tilde{\sigma}_{i 3}^{V}(t) d \tilde{W}_{2 n+i}(t)
\end{array}
$$

By setting $t=T$ in (2.38) and (2.39), we obtain the risk-neutral dynamics of the short rate and short rate spread as

$$
\begin{aligned}
r(t, \mathbb{V}) & =f(0, t)+\sum_{i=1}^{n} \int_{0}^{t} \tilde{\sigma}_{i}^{f}\left(u, t, V_{i}\right) \int_{u}^{t} \tilde{\sigma}_{i}^{f}\left(u, s, V_{i}\right) d s d u+\sum_{i=1}^{n} \int_{0}^{t} \tilde{\sigma}_{i}^{f}\left(u, t, V_{i}\right) d \tilde{W}_{i}(u), \quad(2.42) \\
c(t, \mathbb{V}) & =\lambda(0, t)+\sum_{i=1}^{2 n} \int_{0}^{t} \tilde{\sigma}_{i}^{\lambda}\left(u, t, V_{i}\right) \int_{u}^{t} \tilde{\sigma}_{i}^{\lambda}\left(u, s, V_{i}\right) d s d u+\sum_{i=1}^{n} \int_{0}^{t} \tilde{\sigma}_{i}^{\lambda}\left(u, t, V_{i}\right) \int_{u}^{t} \tilde{\sigma}_{i}^{f}\left(u, s, V_{i}\right) d s d u \\
& +\sum_{i=1}^{n} \int_{0}^{t} \tilde{\sigma}_{i}^{f}\left(u, t, V_{i}\right) \int_{u}^{t} \tilde{\sigma}_{i}^{\lambda}\left(u, s, V_{i}\right) d s d u+\sum_{i=1}^{2 n} \int_{0}^{t} \tilde{\sigma}_{i}^{\lambda}\left(u, t, V_{i}\right) d \tilde{W}_{i}(u) .
\end{aligned}
$$

It follows from (2.34) that under the risk-neutral measure, the short term spread is the product of the market price of jump risk, the jump/default intensity and the loss given default, that is,

$$
c(t, \mathbb{V})=q(t) \psi(t) h(t)=q(t) \tilde{h}(t)
$$


Proposition 2.1 The defaultable bond price can be expressed as

$$
P^{d}(t, T, \mathbb{V})=\mathbb{1}_{\{\tau>t\}} \tilde{\mathbb{E}}\left[e^{-\int_{t}^{T}(r(s, \mathbb{V})+\tilde{h}(s)) d s} \mid \mathcal{F}_{t}^{W}\right]
$$

Proof: Follows from Lando (1998), see Appendix A.

Under general volatility functions, the defaultable forward rate curve (2.40) is nonMarkovian, in general, thereby leading to computational complexity when we need to consider derivative pricing. However in the next section, we propose certain volatility structures that guarantee that the defaultable HJM admits finite dimensional realisations.

\section{A Markovian Defaultable Term Structure Model}

A particular specification of these volatility functions allow us to transform the original non-Markovian structure to Markovian form in line with earlier works in the stochastic volatility, but default-free setting of Chiarella and Kwon (2001) and Björk et al. (2004).

Empirical evidence as given in Collin-Dufresne and Goldstein (2002) and Trolle and Schwartz (2009) suggests that interest rate volatility is stochastic and contains unspanned factors. Bedendo, Cathcart and El-Jahel (2007) demonstrate that the term structure of credit spreads possess hump-shaped curves. In addition, it was noted in Berndt et al. (2010) that the presence of hump volatility improves both the performance of the model and its fit to market data.

Assumption 3.1 For $1 \leq i \leq n$, the volatility functions are of the form

$$
\begin{aligned}
\sigma_{i}^{f}\left(t, T, V_{i}\right) & =\left[a_{0 i}+a_{1 i}(T-t)\right] \sqrt{r(t)} \sqrt{V_{i}(t)} e^{-\kappa_{i}^{f}(T-t)}, \\
\sigma_{i}^{\lambda}\left(t, T, V_{i}\right) & =\left[b_{0 i}+b_{1 i}(T-t)\right] \sqrt{c(t)} \sqrt{V_{i}(t)} e^{-\kappa_{i}^{\lambda}(T-t)},
\end{aligned}
$$

where $\kappa_{i}^{f}, \kappa_{i}^{\lambda}, a_{0 i}, a_{1 i}, b_{0 i}$ and $b_{1 i}$ are constants.

This class of volatility functions gives rise to a high degree of flexibility in modelling the wide range of shapes of the yield curve by virtue of the polynomial in the deterministic part. These volatility specifications are level dependent and involve unspanned stochastic volatility factors. In addition, the specification allows for hump-shaped shocks that are essential in matching interest rate derivatives empirically. 
Proposition 3.1 Under the volatility specification for the default-free forward rate and forward credit spread as specified in Assumption 3.1, the default-free forward rate $f(t, T)$ is expressed as

$$
f(t, T, \mathbb{V})=f(0, T)+\sum_{i=1}^{n} B_{x_{1 i}}(T-t) x_{1 i}(t)+\sum_{i=1}^{n} \sum_{j=1}^{6} B_{\Phi_{j i}}(T-t) \Phi_{j i}(t),
$$

and the forward credit spread $\lambda(t, T)$ is expressed as

$$
\lambda(t, T, \mathbb{V})=\lambda(0, T)+\sum_{i=1}^{n} \sum_{j=2}^{3} B_{x_{j i}}(T-t) x_{j i}(t)+\sum_{i=1}^{n} \sum_{j=7}^{20} B_{\Phi_{j i}}(T-t) \Phi_{j i}(t) .
$$

Thus, the defaultable forward rate $f^{d}(t, T)$ is expressed as

$$
f^{d}(t, T, \mathbb{V})=f^{d}(0, T)+\sum_{i=1}^{n} \sum_{j=1}^{3} B_{x_{j i}}(T-t) x_{j i}(t)+\sum_{i=1}^{n} \sum_{j=1}^{20} B_{\Phi_{j i}}(T-t) \Phi_{j i}(t),
$$

where

$$
\left\{\begin{aligned}
B_{x_{1 i}}(T-t)= & {\left[a_{0 i}+a_{1 i}(T-t)\right] e^{-\kappa_{i}^{f}(T-t)}, } \\
B_{x_{2 i}}(T-t)= & z_{i}^{\lambda_{1}}\left[b_{0 i}+b_{1 i}(T-t)\right] e^{-\kappa_{i}^{\lambda}(T-t),} \\
B_{x_{3 i}}(T-t)= & z_{i}^{\lambda_{2}}\left[b_{0 i}+b_{1 i}(T-t)\right] e^{-\kappa_{i}^{\lambda}(T-t)}, \\
B_{\Phi_{1 i}}(T-t)= & z_{i}^{f_{1}} a_{1 i} e^{-\kappa_{i}^{f}(T-t)}, \\
B_{\Phi_{2 i}}(T-t)= & \frac{a_{1 i}}{\kappa_{i}^{f}}\left(\frac{1}{\kappa_{i}^{f}}+\frac{a_{0 i}}{a_{1 i}}\right)\left[a_{0 i}+a_{1 i}(T-t)\right] e^{-\kappa_{i}^{f}(T-t),} \\
B_{\Phi_{3 i}}(T-t)= & -\left[\frac{a_{1 i} a_{1 i}}{\kappa_{i}^{f}}\left(\frac{1}{\kappa_{i}^{f}}+\frac{a_{0 i}}{a_{1 i}}\right)\right. \\
& \left.+\frac{a_{1 i}}{\kappa_{i}^{f}}\left(\frac{a_{1 i}}{\kappa_{i}^{f}}+2 a_{0 i}\right)(T-t)+\frac{\left(a_{1 i}\right)^{2}}{\kappa_{i}^{f}}(T-t)^{2}\right] e^{-2 \kappa_{i}^{f}(T-t),} \\
B_{\Phi_{4 i}}(T-t)= & \frac{\left(a_{1 i}\right)^{2}}{\kappa_{i}^{f}}\left(\frac{1}{\kappa_{i}^{f}}+\frac{a_{0 i}}{a_{1 i}}\right) e^{-\kappa_{i}^{f}(T-t),} \\
B_{\Phi_{5 i}}(T-t)= & -\frac{a_{1 i}}{\kappa_{i}^{f}}\left[\frac{a_{1 i}}{\kappa_{i}^{f}}+2 a_{0 i}+2 a_{1 i}(T-t)\right] e^{-2 \kappa_{i}^{f}(T-t),} \\
B_{\Phi_{6 i}}(T-t)= & -\frac{\left(a_{1 i}\right)^{2}}{\kappa_{i}^{f}} e^{-2 \kappa_{i}^{f}(T-t)},
\end{aligned}\right.
$$




$$
\begin{aligned}
& \left\{\begin{aligned}
B_{\Phi_{7 i}}(T-t)= & z_{i}^{\lambda_{1}}\left[\frac{a_{0 i} b_{0 i}}{\kappa_{i}^{f}}+\frac{a_{1 i} b_{0 i}}{\left(\kappa_{i}^{f}\right)^{2}}+\left(\frac{a_{0 i} b_{1 i}}{\kappa_{i}^{f}}+\frac{a_{1 i} b_{1 i}}{\left(\kappa_{i}^{f}\right)^{2}}\right)(T-t)\right] e^{-\kappa_{i}^{\lambda}(T-t)}, \\
B_{\Phi_{8 i}}(T-t)=- & z_{i}^{\lambda_{1}}\left[\frac{a_{0 i} b_{0 i}}{\kappa_{i}^{f}}+\frac{a_{1 i} b_{0 i}}{\left(\kappa_{i}^{f}\right)^{2}}+\left(\frac{a_{1 i} b_{0 i}}{\kappa_{i}^{f}}+\frac{a_{0 i} b_{1 i}}{\kappa_{i}^{f}}+\frac{a_{1 i} b_{1 i}}{\left(\kappa_{i}^{f}\right)^{2}}\right)(T-t)\right. \\
& \left.\quad+\frac{a_{1 i} b_{1 i}}{\kappa_{i}^{f}}(T-t)^{2}\right] e^{-\left(\kappa_{i}^{f}+\kappa_{i}^{\lambda}\right)(T-t)}, \\
B_{\Phi_{9 i}}(T-t)= & z_{i}^{\lambda_{1}}\left(\frac{a_{0 i} b_{0 i}}{\kappa_{i}^{f}}+\frac{a_{1 i} b_{1 i}}{\left(\kappa_{i}^{f}\right)^{2}}\right) e^{-\kappa_{i}^{\lambda}(T-t)}, \\
B_{\Phi_{10 i}}(T-t)= & -z_{i}^{\lambda_{1}}\left[\frac{a_{1 i} b_{0 i}}{\kappa_{i}^{f}}+\frac{a_{0 i} b_{1 i}}{\kappa_{i}^{f}}+\frac{a_{1 i} b_{1 i}}{\left(\kappa_{i}^{f}\right)^{2}}-2 \frac{a_{1 i} b_{1 i}}{\kappa_{i}^{f}}(T-t)\right] e^{-\left(\kappa_{i}^{f}+\kappa_{i}^{\lambda}\right)(T-t)} \\
B_{\Phi_{11 i}}(T-t)= & -z_{i}^{\lambda_{1}} \frac{a_{1 i} b_{1 i}}{\kappa_{i}^{f}} e^{-\left(\kappa_{i}^{f}+\kappa_{i}^{\lambda}\right)(T-t)}, \\
B_{\Phi_{12 i}}(T-t)= & z_{i}^{\lambda_{1}}\left[\frac{a_{0 i} b_{0 i}}{\kappa_{i}^{\lambda}}+\frac{a_{0 i} b_{1 i}}{\left(\kappa_{i}^{\lambda}\right)^{2}}+\left(\frac{a_{1 i} b_{0 i}}{\kappa_{i}^{\lambda}}+\frac{a_{1 i} b_{1 i}}{\left(\kappa_{i}^{\lambda}\right)^{2}}\right)(T-t)\right] e^{-\kappa_{i}^{f}(T-t)}, \\
B_{\Phi_{13 i}}(T-t)= & z_{i}^{\lambda_{1}}\left(\frac{a_{0 i} b_{0 i}}{\kappa_{i}^{\lambda}}+\frac{a_{1 i} b_{1 i}}{\left(\kappa_{i}^{\lambda}\right)^{2}}\right) e^{-\kappa_{i}^{f}(T-t)}
\end{aligned}\right. \\
& \left\{\begin{array}{l}
B_{\Phi_{14 i}}(T-t)=\frac{b_{1 i}}{\kappa_{i}^{\lambda}}\left(\frac{1}{\kappa_{i}^{\lambda}}+\frac{b_{0 i}}{b_{1 i}}\right)\left[b_{0 i}+b_{1 i}(T-t)\right] e^{-\kappa_{i}^{\lambda}(T-t),} \\
B_{\Phi_{15 i}}(T-t)=\left[\frac{b_{1 i} b_{1 i}}{\kappa_{i}^{\lambda}}\left(\frac{1}{\kappa_{i}^{\lambda}}+\frac{b_{0 i}}{b_{1 i}}\right)+\frac{b_{1 i}}{\kappa_{i}^{\lambda}}\left(\frac{b_{1 i}}{\kappa_{i}^{\lambda}}+2 b_{0 i}\right)(T-t)+\frac{\left(b_{1 i}\right)^{2}}{\kappa_{i}^{\lambda}}(T-t)^{2}\right] e^{-2 \kappa_{i}^{\lambda}(T-t),} \\
B_{\Phi_{16 i}}(T-t)=\frac{\left(b_{1 i}\right)^{2}}{\kappa_{i}^{\lambda}}\left(\frac{1}{\kappa_{i}^{\lambda}}+\frac{b_{0 i}}{b_{1 i}}\right) e^{-\kappa_{i}^{\lambda}(T-t)}, \\
B_{\Phi_{17 i}}(T-t)=-\frac{b_{1 i}}{\kappa_{i}^{\lambda}}\left[\frac{b_{1 i}}{\kappa_{i}^{\lambda}}+2 b_{0 i}+2 b_{1 i}(T-t)\right] e^{-2 \kappa_{i}^{\lambda}(T-t),} \\
B_{\Phi_{18 i}}(T-t)=-\frac{\left(b_{1 i}\right)^{2}}{\kappa_{i}^{\lambda}} e^{-2 \kappa_{i}^{\lambda}(T-t)}, \\
B_{\Phi_{19 i}}(T-t)=z_{i}^{\lambda} b_{1 i} e^{-\kappa_{i}^{\lambda}(T-t)}, \\
B_{\Phi_{20 i}}(T-t)=z_{i}^{\lambda_{2}} b_{1 i} e^{-\kappa_{i}^{\lambda}(T-t)},
\end{array}\right.
\end{aligned}
$$

and the state variables $x_{j i}(t)$ and $\Phi_{j i}(t)$ satisfy the stochastic differential equations given in Corollary 3.2 whereas the correlations $z_{i}^{f_{1}}, z_{i}^{\lambda_{1}}, z_{i}^{\lambda_{2}}$ are defined in (2.22).

Proof: See Appendix B for technical details.

Corollary 3.2 The state variables $x_{j i}(t)$ and $\Phi_{j i}(t)$ satisfy the stochastic differential 
equations

$$
\left\{\begin{array}{l}
d x_{1 i}(t)=-\kappa_{i}^{f} x_{1 i}(t) d t+\sqrt{r(t) V_{i}(t)} d \tilde{W}_{i}(t), \\
d x_{2 i}(t)=-\kappa_{i}^{\lambda} x_{2 i}(t) d t+\sqrt{c(t) V_{i}(t)} d \tilde{W}_{i}(t), \\
d x_{3 i}(t)=-\kappa_{i}^{\lambda} x_{3 i}(t) d t+\sqrt{c(t) V_{i}(t)} d \tilde{W}_{n+i}(t), \\
d \Phi_{1 i}(t)=\left[x_{1 i}(t)-\kappa_{i}^{f} \Phi_{1 i}(t)\right] d t, \quad d \Phi_{2 i}(t)=\left[r(t) V_{i}(t)-\kappa_{i}^{f} \Phi_{2 i}(t)\right] d t, \\
d \Phi_{3 i}(t)=\left[r(t) V_{i}(t)-2 \kappa_{i}^{f} \Phi_{3 i}(t)\right] d t, \quad d \Phi_{4 i}(t)=\left[\Phi_{2 i}(t)-\kappa_{i}^{f} \Phi_{4 i}(t)\right] d t, \\
d \Phi_{5 i}(t)=\left[\Phi_{3 i}(t)-2 \kappa_{i}^{f} \Phi_{5 i}(t)\right] d t, \quad d \Phi_{6 i}(t)=\left[2 \Phi_{5 i}(t)-2 \kappa_{i}^{f} \Phi_{6 i}(t)\right] d t, \\
d \Phi_{7 i}(t)=\left[V_{i}(t) \sqrt{r(t) c(t)}-\kappa_{i}^{\lambda} \Phi_{7 i}(t)\right] d t, \quad d \Phi_{8 i}(t)=\left[V_{i}(t) \sqrt{r(t) c(t)}-\left(\kappa_{i}^{f}+\kappa_{i}^{\lambda}\right) \Phi_{8 i}(t)\right] d t \\
d \Phi_{9 i}(t)=\left[\Phi_{9 i}(t)-\kappa_{i}^{\lambda} \Phi_{9 i}(t)\right] d t, \quad d \Phi_{10 i}(t)=\left[\Phi_{10 i}(t)-\left(\kappa_{i}^{f}+\kappa_{i}^{\lambda}\right) \Phi_{10 i}(t)\right] d t, \\
d \Phi_{11 i}(t)=\left[2 \Phi_{12 i}(t)-\left(\kappa_{i}^{f}+\kappa_{i}^{\lambda}\right) \Phi_{11 i}(t)\right] d t, \quad d \Phi_{12 i}(t)=\left[V_{i}(t) \sqrt{r(t) c(t)}-\kappa_{i}^{f} \Phi_{12 i}(t)\right] d t, \\
d \Phi_{13 i}(t)=\left[\Phi_{14 i}(t)-\kappa_{i}^{f} \Phi_{13 i}(t)\right] d t, \quad d \Phi_{14 i}(t)=\left[c(t) V_{i}(t)-\kappa_{i}^{\lambda} \Phi_{14 i}(t)\right] d t, \\
d \Phi_{15 i}(t)=\left[c(t) V_{i}(t)-2 \kappa_{i}^{\lambda} \Phi_{15 i}(t)\right] d t, \quad d \Phi_{16 i}(t)=\left[\Phi_{16 i}(t)-\kappa_{i}^{\lambda} \Phi_{16 i}(t)\right] d t, \\
d \Phi_{17 i}(t)=\left[\Phi_{15 i}(t)-2 \kappa_{i}^{\lambda} \Phi_{17 i}(t)\right] d t, \quad d \Phi_{18 i}(t)=\left[2 \Phi_{17 i}(t)-2 \kappa_{i}^{\lambda} \Phi_{18 i}(t)\right] d t, \\
d \Phi_{19 i}(t)=\left[x_{2 i}(t)-\kappa_{i}^{\lambda} \Phi_{19 i}(t)\right] d t, \quad d \Phi_{20 i}(t)=\left[x_{3 i}(t)-\kappa_{i}^{\lambda} \Phi_{20 i}(t)\right] d t,
\end{array}\right.
$$

subject to the initial conditions $x_{j i}(0)=\Phi_{j i}(0)=0$ for $i=1, \ldots, n$ and $j=1, \ldots, 20$.

Proof: Take the stochastic differential of $(\underline{B .4}),(\underline{B} .10)$ and $(\mathrm{B} .12)$ in Appendix B to obtain the stochastic differential equations. Note that the system in Corollary 3.2 needs to be augmented by the stochastic differential equations for the volatility functions $V_{i}(t)$ that satisfy the SDEs (2.41).

Corollary 3.3 The short rate and the short term credit spread processes can be expressed as

$$
\begin{aligned}
& r(t, \mathbb{V})=f(0, t)+\sum_{i=1}^{n} \alpha_{1 i} x_{1 i}(t)+\sum_{i=1}^{n} \sum_{j=1}^{6} \beta_{j i} \Phi_{j i}(t), \\
& c(t, \mathbb{V})=\lambda(0, t)+\sum_{i=1}^{n} \sum_{j=2}^{3} \alpha_{j i} x_{j i}(t)+\sum_{i=1}^{n} \sum_{j=7}^{20} \beta_{j i} \Phi_{j i}(t),
\end{aligned}
$$

where $\alpha_{j i}=B_{x_{j i}}(0)$ and $\beta_{j i}=B_{\Phi_{j i}}(0)$.

Proof: By using (3.3) and $r(t, \mathbb{V})=f(t, t, \mathbb{V})$, as well as (3.4) and $c(t, \mathbb{V})=\lambda(t, t, \mathbb{V})$. 
Proposition 3.4 The price of a default-free bond can be expressed in the exponential affine form

$$
P(t, T, \mathbb{V})=\frac{P(0, T)}{P(0, t)} \exp \left(-\sum_{i=1}^{n} D_{x_{1 i}}(T-t) x_{1 i}(t)-\sum_{i=1}^{n} \sum_{j=1}^{6} D_{\Phi_{j i}}(T-t) \Phi_{j i}(t)\right)
$$

The defaultable zero-coupon bond $P^{d}(t, T, \mathbb{V})=\mathcal{R}(t) \bar{P}^{d}(t, T, \mathbb{V})$ is exponential affine with

$$
\bar{P}^{d}(t, T, \mathbb{V})=\frac{\bar{P}^{d}(0, T)}{\bar{P}^{d}(0, t)} \exp \left(-\sum_{i=1}^{n} \sum_{j=1}^{3} D_{x_{j i}}(T-t) x_{j i}(t)-\sum_{i=1}^{n} \sum_{j=1}^{20} D_{\Phi_{j i}}(T-t) \Phi_{j i}(t)\right),
$$

where $x_{j i}(t)$ and $\Phi_{j i}(t)$ are specified in Corollary 3.2 and the deterministic functions $D_{x_{j i}}(T-t)$ and $D_{\Phi_{j i}}(T-t)$ are given by

$$
\begin{aligned}
& \left\{\begin{array}{l}
D_{x_{1 i}}(T-t)=\frac{z_{i}^{f_{1}}}{\left(\kappa_{i}^{f}\right)^{2}}\left[a_{0 i} \kappa_{i}^{f}+a_{1 i}-e^{-\kappa_{i}^{f}(T-t)}\left(a_{0 i} \kappa_{i}^{f}+a_{1 i}+a_{1 i} \kappa_{i}^{f}(T-t)\right)\right], \\
D_{x_{2 i}}(T-t)=\frac{z_{i}^{\lambda_{1}}}{\left(\kappa_{i}^{\lambda}\right)^{2}}\left[a_{0 i} \kappa_{i}^{\lambda}+a_{1 i}-e^{-\kappa_{i}^{\lambda}(T-t)}\left(a_{0 i} \kappa_{i}^{\lambda}+a_{1 i}+a_{1 i} \kappa_{i}^{\lambda}(T-t)\right)\right], \\
D_{x_{3 i}}(T-t)=\frac{z_{i}^{\lambda_{2}}}{\left(\kappa_{i}^{\lambda}\right)^{2}}\left[a_{0 i} \kappa_{i}^{\lambda}+a_{1 i}-e^{-\kappa_{i}^{\lambda}(T-t)}\left(a_{0 i} \kappa_{i}^{\lambda}+a_{1 i}+a_{1 i} \kappa_{i}^{\lambda}(T-t)\right)\right],
\end{array}\right. \\
& \left\{\begin{aligned}
D_{\Phi_{1 i}}(T-t) & =\frac{z_{i}^{f_{1}} a_{1 i}}{\kappa_{i}^{f}}\left(1-e^{-\kappa_{i}^{f}(T-t)}\right), \\
D_{\Phi_{2 i}}(T-t) & =\left(\frac{a_{1 i}}{\kappa_{i}^{f}}\right)^{2}\left(\frac{1}{\kappa_{i}^{f}}+\frac{a_{0 i}}{a_{1 i}}\right)\left[\left(\frac{1}{\kappa_{i}^{f}}+\frac{a_{0 i}}{a_{1 i}}\right)\left(e^{-\kappa_{i}^{f}(T-t)}-1\right)+(T-t) e^{-\kappa_{i}^{f}(T-t)}\right], \\
D_{\Phi_{3 i}}(T-t) & =-\left(\frac{a_{1 i}}{\left(\kappa_{i}^{f}\right)^{2}}\right)\left[\left(\frac{a_{1 i}}{2\left(\kappa_{i}^{f}\right)^{2}}+\frac{a_{0 i}}{\kappa_{i}^{f}}+\frac{\left(a_{0 i}\right)^{2}}{2 a_{1 i}}\right)\left(e^{-2 \kappa_{i}^{f}(T-t)}-1\right)\right. \\
& +\left(\frac{a_{1 i}}{\kappa_{i}^{f}}+a_{0 i}\right)(T-t) e^{-2 \kappa_{i}^{f}(T-t)}+\frac{a_{1 i}}{2}(T-t)^{2} e^{\left.-2 \kappa_{i}^{f}(T-t)\right]} \\
D_{\Phi_{4 i}}(T-t) & =\left(\frac{a_{1 i}}{\kappa_{i}^{f}}\right)^{2}\left(\frac{1}{\kappa_{i}^{f}}+\frac{a_{0 i}}{a_{1 i}}\right)\left(e^{-\kappa_{i}^{f}(T-t)}-1\right), \\
D_{\Phi_{5 i}}(T-t) & =-\left(\frac{a_{1 i}}{\left(\kappa_{i}^{f}\right)^{2}}\right)\left[\left(\frac{a_{1 i}}{\kappa_{i}^{f}}+a_{0 i}\right)\left(e^{-2 \kappa_{i}^{f}(T-t)}-1\right)+a_{1 i}(T-t) e^{\left.-2 \kappa_{i}^{f}(T-t)\right]}\right. \\
D_{\Phi_{6 i}}(T-t) & =-\frac{1}{2}\left(\frac{a_{1 i}}{\kappa_{i}^{f}}\right)^{2}\left(e^{-2 \kappa_{i}^{f}(T-t)}-1\right),
\end{aligned}\right.
\end{aligned}
$$




$$
\begin{aligned}
& \int D_{\Phi_{7 i}}(T-t)=\frac{z_{i}^{\lambda_{1}}}{\kappa_{i}^{f} \kappa_{i}^{\lambda}}\left[\left(a_{0 i}+\frac{a_{1 i}}{k_{i}^{f}}\right) b_{1 i}(T-t) e^{-\kappa_{i}^{\lambda}(T-t)}\right], \\
& D_{\Phi_{8 i}}(T-t)=-\frac{z_{i}^{\lambda_{1}}}{\kappa_{i}^{f}+\kappa_{i}^{\lambda}}\left\{\left(\frac{a_{0 i} b_{0 i}}{\kappa_{i}^{f}}+\frac{a_{1 i} b_{0 i}}{\left(\kappa_{i}^{f}\right)^{2}}\right)\left(e^{-\left(\kappa_{i}^{f}+\kappa_{i}^{\lambda}\right)(T-t)}-1\right)\right. \\
& +\frac{1}{\kappa_{i}^{f}+\kappa_{i}^{\lambda}}\left(\frac{a_{1 i} b_{0 i}}{\kappa_{i}^{f}}+\frac{a_{0 i} b_{1 i}}{\kappa_{i}^{f}}+\frac{a_{1 i} b_{1 i}}{\left(\kappa_{i}^{f}\right)^{2}}\right)\left[1-e^{-\left(\kappa_{i}^{f}+\kappa_{i}^{\lambda}\right)(T-t)}-\left(\kappa_{i}^{f}+\kappa_{i}^{\lambda}\right)(T-t) e^{-\left(\kappa_{i}^{f}+\kappa_{i}^{\lambda}\right)(T-t)}\right] \\
& \left.+\frac{1}{\kappa_{i}^{f}+\kappa_{i}^{\lambda}}\left(\frac{a_{1 i} b_{1 i}}{\kappa_{i}^{f}}\right)\left[2-e^{-\left(\kappa_{i}^{f}+\kappa_{i}^{\lambda}\right)(T-t)}\left(2-\left(\kappa_{i}^{f}+\kappa_{i}^{\lambda}\right)(T-t)\left(2-\left(\kappa_{i}^{f}+\kappa_{i}^{\lambda}\right)(T-t)\right)\right)\right]\right\}, \\
& D_{\Phi_{9 i}}(T-t)=\frac{z_{i}^{\lambda_{1}}}{\kappa_{i}^{\lambda}}\left(\frac{a_{0 i} b_{0 i}}{\kappa_{i}^{f}}+\frac{a_{1 i} b_{1 i}}{\left(\kappa_{i}^{f}\right)^{2}}\right)\left(1-e^{-\kappa_{i}^{\lambda}(T-t)}\right), \\
& D_{\Phi_{10 i}}(T-t)=-\frac{z_{i}^{\lambda_{1}}}{\kappa_{i}^{f}\left(\kappa_{i}^{f}+\kappa_{i}^{\lambda}\right)}\left\{\left(\left(a_{0 i} b_{1 i}+\frac{a_{1 i} b_{1 i}}{\kappa_{i}^{f}}\right)\left(b_{0 i}-2 \frac{b_{1 i}}{\kappa_{i}^{f}+\kappa_{i}^{\lambda}}\right)\left(1-e^{-\left(\kappa_{i}^{f}+\kappa_{i}^{\lambda}\right)(T-t)}\right)\right.\right. \\
& \left.+2 b_{1 i}(T-t) e^{-\left(\kappa_{i}^{f}+\kappa_{i}^{\lambda}\right)(T-t)}\right\} \\
& D_{\Phi_{11 i}}(T-t)=-\frac{z_{i}^{\lambda_{1}}}{\left(\kappa_{i}^{f}+\kappa_{i}^{\lambda}\right)} \frac{a_{1 i} b_{1 i}}{\kappa_{i}^{f}}\left(1-e^{-\left(\kappa_{i}^{f}+\kappa_{i}^{\lambda}\right)(T-t)}\right), \\
& D_{\Phi_{12 i}}(T-t)=\frac{z_{i}^{\lambda_{1}}}{\kappa_{i}^{f} \kappa_{i}^{\lambda}}\left[\left(b_{0 i}+\frac{b_{1 i}}{k_{i}^{f}}\right) a_{1 i}(T-t) e^{-\kappa_{i}^{f}(T-t)}\right] \text {, } \\
& D_{\Phi_{13 i}}(T-t)=\frac{z_{i}^{\lambda_{1}}}{\kappa_{i}^{f}}\left(\frac{a_{0 i} b_{0 i}}{\kappa_{i}^{\lambda}}+\frac{a_{1 i} b_{1 i}}{\left(\kappa_{i}^{\lambda}\right)^{2}}\right)\left(1-e^{-\kappa_{i}^{f}(T-t)}\right),
\end{aligned}
$$

and finally we have

$$
\left\{\begin{aligned}
D_{\Phi_{14 i}}(T-t) & =\left(\frac{b_{1 i}}{\kappa_{i}^{\lambda}}\right)^{2}\left(\frac{1}{\kappa_{i}^{\lambda}}+\frac{b_{0 i}}{b_{1 i}}\right)\left[\left(\frac{1}{\kappa_{i}^{\lambda}}+\frac{b_{0 i}}{b_{1 i}}\right)\left(e^{-\kappa_{i}^{\lambda}(T-t)}-1\right)+(T-t) e^{-\kappa_{i}^{\lambda}(T-t)}\right], \\
D_{\Phi_{15 i}}(T-t) & =-\left(\frac{b_{1 i}}{\left(\kappa_{i}^{\lambda}\right)^{2}}\right)\left[\left(\frac{b_{1 i}}{2\left(\kappa_{i}^{\lambda}\right)^{2}}+\frac{b_{0 i}}{\kappa_{i}^{\lambda}}+\frac{\left(b_{0 i}\right)^{2}}{2 b_{1 i}}\right)\left(e^{-2 \kappa_{i}^{\lambda}(T-t)}-1\right)\right. \\
+ & \left(\frac{b_{1 i}}{\kappa_{i}^{\lambda}}+b_{0 i}\right)(T-t) e^{-2 \kappa_{i}^{\lambda}(T-t)}+\frac{b_{1 i}}{2}(T-t)^{2} e^{\left.-2 \kappa_{i}^{\lambda}(T-t)\right]}, \\
D_{\Phi_{16 i}}(T-t) & =\left(\frac{b_{1 i}}{\kappa_{i}^{\lambda}}\right)^{2}\left(\frac{1}{\kappa_{i}^{\lambda}}+\frac{b_{0 i}}{b_{1 i}}\right)\left(e^{-\kappa_{i}^{\lambda}(T-t)}-1\right), \\
D_{\Phi_{17 i}}(T-t) & =-\left(\frac{b_{1 i}}{\left(\kappa_{i}^{\lambda}\right)^{2}}\right)\left[\left(\frac{b_{1 i}}{\kappa_{i}^{\lambda}}+b_{0 i}\right)\left(e^{-2 \kappa_{i}^{\lambda}(T-t)}-1\right)+b_{1 i}(T-t) e^{\left.-2 \kappa_{i}^{\lambda}(T-t)\right]}\right. \\
D_{\Phi_{18 i}}(T-t) & =-\frac{1}{2}\left(\frac{b_{1 i}}{\kappa_{i}^{\lambda}}\right)^{2}\left(e^{-2 \kappa_{i}^{\lambda}(T-t)}-1\right), \quad D_{\Phi_{19 i}}(T-t)=\frac{z_{i}^{\lambda_{1}} b_{1 i}}{\kappa_{i}^{\lambda}}\left(1-e^{-\kappa_{i}^{\lambda}(T-t)}\right), \\
D_{\Phi_{20 i}}(T-t) & =\frac{z_{i}^{\lambda} b_{1 i}}{\kappa_{i}^{\lambda}}\left(1-e^{-\kappa_{i}^{\lambda}(T-t)}\right) .
\end{aligned}\right.
$$

Proof: See Appendix C for the technical details.

Proposition 3.4 is the main theoretical contribution of the paper. The default-free bond price can be expressed in terms of $8 n(=7 n+n)$ state variables while the defaultable bond price is expressed in terms of $24 n(=23 n+n)$ state variables, where $n$ of the state variables are associated with $n$ stochastic volatility variables. Both default-free and defaultable bond prices are exponential affine functions of these state variables and the $7 n$ state variables driving the default-free bond prices constitute a common set for 
both default-free and defaultable bond prices. Even though the dimension of the state space is relatively large, the driving sources of uncertainty of the entire state space are only $3 n$. Thus assessing the model's flexibility and suitability for estimation or calibration applications, this Markovian defautlable model can be moderately demanding computationally.

The proposed model can be easily adjusted to accommodate exponentially decaying volatility functional forms for the forward rates and credit spreads resulting in a Markovian system with a lower dimension state space as has been shown in Chiarella, Maina and Nikitopolous-Sklibosios (2010). We also note that we could obtain finite dimensional realisations by expressing the state variables as a linear combination of fixed tenor forward rates, following the lines of Chiarella and Kwon (2003) in the default-free framework and Chiarella et al. (2007) in the defaultable HJM framework.

The proposed defaultable term structure model will be used next to price analytically credit derivatives, for instance, credit default swaps and swaptions. Maina (2011) demonstrates that by excluding the level dependency on the forward rate and credit spread volatility specifications of Assumption 3.1, semi-closed solutions for options on defaultable bonds can be derived. Pricing of options on defaultable bonds under the broad volatility specifications (3.1) can only be performed numerically via Monte Carlo simulations, see for instance Chiarella, Fanelli and Musti (2011), where a defaultable term structure model is developed with level dependent volatilities, but without stochastic volatility.

\section{Pricing of Credit Default Swaps and Swaptions}

In this section, we derive pricing formulas for single-name credit default swaps (CDS) and default swaptions in the absence of counterparty risk. The possible extension to incorporate counterparty risk is discussed in Section 4.2 ,

Default is modelled as the first jump of a Cox process, for instance $\tau=\tau_{1}$ as defined in Section 2 and the subfiltration $\mathcal{F}^{\tau}$ is

$$
\left(\mathcal{F}_{t}^{\tau}\right)_{t \geq 0}=\{\sigma(\tau>s: 0 \leq s \leq t)\}_{t \geq 0}
$$

namely the $\sigma$-algebra generated by $\tau$. In the following sections, we assume that the

filtration defined in Section 2 is given by $\mathcal{F}_{t}=\mathcal{F}_{t}^{W} \vee \mathcal{F}_{t}^{\tau}, t \geq 0$. The survival probability is given by $\mathbb{Q}\left(t<\tau \mid \mathcal{F}_{t}^{W}\right)$. 


\subsection{CDS with no Counterparty Risk}

A CDS contract involves three parties, the insurance buyer or insured party, the insurance seller or insurer and the reference obligor. A CDS contract with maturity $T$ allows the insured party to receive protection, up to time $T$, from the insurer against default of the reference obligor. The insured party pays a regular fee $\pi$ (premium) to the insurer in return for a protection payment upon default. In the absence of counterparty risk, the default time $\tau<T$ represents the time at which the reference obligor fails to make the required payments on the structured reference bonds. When $t<\tau \leq T$, then the insurer has to make a protection payment $(1-\mathcal{R})$ at default time $\tau$, where $\mathcal{R}$ is the recovery rate prevailing at the default time $\tau$, which we assume to be given 2 Then the value of the protection leg, under the physical settlement assumption 3 at $t<\tau$ is

$$
W_{p r t}(t)=e^{-\int_{t}^{\tau} r(s) d s}(1-\mathcal{R}) \mathbb{1}_{\{t<\tau \leq T\}} .
$$

The insured party pays the premium $\pi$ at times $t_{i}, i=1,2, \ldots, N$ until either the contract maturity $t_{N}=T$, if no default occurs, or until default, if $\tau \leq T$. By setting $\delta_{i}=t_{i-1}-t_{i}$, then the value at time $t<t_{1}$ of the premium leg, including the accrual payment for the fraction of time in which default occurs, is given by

$$
W_{\text {prm }}(t)=\pi \sum_{i=1}^{N} \delta_{i} e^{-\int_{t}^{t_{i}} r(s) d s} \mathbb{1}_{\left\{\tau>t_{i}\right\}}+\pi\left(\tau-t_{\tau-1}\right) e^{-\int_{t}^{\tau} r(s) d s} \mathbb{1}_{\{t<\tau<T\}},
$$

where $t_{1}$ is the first premium payment date and $t_{\tau-1}$ is the last premium payment date $t_{i}$ before default time $\tau$.

For notational simplicity, we assume that the default intensity (under the risk-neutral measure) is independent of the jump size $\tilde{h}(t, d q)=\tilde{h}(t)$. Under no-arbitrage pricing, the value of the CDS can be expressed under the risk-neutral probability measure as

$$
C D S_{\pi}(t)=\tilde{\mathbb{E}}\left[W_{p r t}(t)-W_{p r m}(t) \mid \mathcal{F}_{t}\right] .
$$

\footnotetext{
${ }^{2}$ We note that although we use $\mathcal{R}$ for the recovery rate of the underlying risky asset, this is not the recovery rate as used in Section 3 In this section, $\mathcal{R}$ has been used to denote recovery in the underlying contract on default of the obligor, whereas in the previous sections there was the specific reference to the underlying defaultable bond.

${ }^{3}$ Default can be settled physically where A and B exchange one of the specified reference bonds at its par value or alternatively as a cash-settlement, which is the common market practice. In this case, several independent dealers are asked to provide quotes on the defaulted bond, and party $B$ pays party $A$ the difference between the average quoted value and the par bond value. In a CDS, the protection buyer is effectively long on a delivery option which gives the buyer the right to deliver the 'cheapest-to-deliver' asset to the protection seller.
} 
The fair premium rate $\tilde{\pi}(t)$, the so called CDS spread, is the rate that will make the value of the CDS equal to zero at inception of the contract.

Proposition 4.1 When the contract is settled (that is, the protection payment is made) immediately on default of the reference obligor, the CDS spread is given by

$$
\tilde{\pi}(t)=\frac{\mathbb{1}_{\{\tau>t\}}(1-\mathcal{R}) \int_{t}^{T} \tilde{\mathbb{E}}\left[\tilde{h}(u) e^{-\int_{t}^{u}(r(s)+\tilde{h}(s)) d s} \mid \mathcal{F}_{t}^{W}\right] d u}{\mathbb{1}_{\{\tau>t\}} \sum_{i=1}^{N} \delta_{i} \bar{P}^{d}\left(t, t_{i}\right)+\tilde{\mathbb{E}}\left[\left(\tau-t_{\tau-1}\right) e^{-\int_{t}^{\tau} r(s) d s} \mathbb{1}_{\{t<\tau<T\}} \mid \mathcal{F}_{t}\right]} .
$$

Proof: See Appendix D.

Instead of allowing the protection payment to be made at default time $\tau$, the protection payment could be deferred to the first premium payment date $t_{i}$ following default time $\left(t_{i}>\tau\right)$. This gives rise to the postponed running CDS whose main advantage is that the absence of accrued-interest term in $\left(\tau-t_{\tau-1}\right)$ ensures that all payments occur at the canonical grid of the $t_{i}$ 's. We then have the following result.

Corollary 4.2 By assuming that the protection payment is postponed to the first premium payment date $t_{i}$ following default time, the CDS spread can be approximated by

$$
\tilde{\pi}(t) \approx \frac{(1-\mathcal{R}) \sum_{i=1}^{N}\left[\bar{P}^{d}\left(t, t_{i-1}\right)-\bar{P}^{d}\left(t, t_{i}\right)\right]}{\sum_{i=1}^{N}\left(t_{i}-t_{i-1}\right) \bar{P}^{d}\left(t, t_{i}\right)} .
$$

Proof: Follows the approach of Brigo and Morini (2005). See also Appendix E,

\subsection{CDS with Counterparty Risk}

To determine the CDS spread in the presence of counterparty risk, the inter-dependent default risk structures between these parties should be considered simultaneously. It was shown in Jarrow and Yu (2001) that a CDS may be significantly overpriced if the default correlation between insurance seller and reference obligor is ignored. Hull and White (2001) argue that if the default correlation is positive, then the default of the counterparty will result in a positive replacement cost for the insured party.

Following Chen and Filipovič (2007), we let $\tau_{1}, \tau_{2}, \tau_{3}$ be the default times of the reference obligor, the insured party and the insurance seller, respectively. The insured party pays the premium $\pi$ at times $t_{i}$ only, given the events that happened in the preceding periods. At time $t_{i}<\tau_{1} \wedge \tau_{2} \wedge \tau_{3}$, the insured party pays to the seller the

\footnotetext{
${ }^{4}$ We use the approximation $e^{-\int_{t}^{t_{i}} r(s) d s} \approx e^{-\int_{t}^{t_{i-1}} r(s) d s}$ made in (E.2) whose error is of a very small order for small values of $\delta$.
} 
fixed rate $\pi$ if no default has taken place. If the reference obligor has defaulted in the period $\left(t_{i-1}, t_{i}\right]$, that is $t_{i-1}<\tau_{1} \leq t_{i}$ and the insured party has not defaulted by time $t_{i-1}, \tau_{2}>t_{i-1}$ and the insurance seller has not yet defaulted by time $t_{i}$ with $\tau_{3}>t_{i}$, then the seller pays $(1-\mathcal{R})$ and the contract terminates. Otherwise if either the insured party or the insurance seller defaults before then, there is no payment and the contract terminates. The protection payment is therefore made only on the occurrence of event $\tau_{1}$ and zero otherwise.

Assuming a postponed running CDS, the value at time $t<t_{1}$ of the premium leg is given by

$$
W_{p r m}^{c p r}(t)=\pi \sum_{i=1}^{N} \delta_{i} e^{-\int_{t}^{t_{i}} r(s) d s} \mathbb{1}_{\left\{\tau_{1} \wedge \tau_{2} \wedge \tau_{3}>t_{i}\right\}}
$$

and similarly, we can express the value of the protection leg as

$$
W_{\text {prt }}^{c p r}(t)=(1-\mathcal{R}) \sum_{i=1}^{N} e^{-\int_{t}^{t_{i}} r(s) d s} \mathbb{1}_{\left\{t_{i-1}<\tau_{1} \leq t_{i}\right\}} \mathbb{1}_{\left\{\tau_{2}>t_{i-1}\right\}} \mathbb{1}_{\left\{\tau_{3}>t_{i}\right\}} .
$$

The fair CDS spread $\tilde{\pi}_{c p r}(t)$, in the presence of counterparty risk, at time $t<t_{1}$ is the fixed rate which guarantees that the value of the CDS is zero, namely,

$$
C D S_{c p r}(t)=\tilde{\mathbb{E}}\left[W_{p r t}^{c p r}(t)-W_{p r m}^{c p r}(t) \mid \mathcal{F}_{t}\right]=0
$$

and is expressed as

$$
\tilde{\pi}_{c p r}(t)=\frac{(1-\mathcal{R}) \sum_{i=1}^{N} \tilde{\mathbb{E}}\left[e^{-\int_{t}^{t_{i}} r(s) d s}\left(\mathbb{1}_{\left\{\tau_{1}>t_{i-1}\right\}}-\mathbb{1}_{\left\{\tau_{1}>t_{i}\right\}}\right) \mathbb{1}_{\left\{\tau_{2}>t_{i-1}\right\}} \mathbb{1}_{\left\{\tau_{3}>t_{i}\right\}} \mid \mathcal{F}_{t}\right]}{\delta \sum_{i=1}^{N} \tilde{\mathbb{E}}\left[e^{-\int_{t}^{t_{i}} r(s) d s} \mathbb{1}_{\left\{\tau_{1} \wedge \tau_{2} \wedge \tau_{3}>t_{i}\right\}} \mid \mathcal{F}_{t}\right]}
$$

The CDS spread (4.10) can be approximated by a ratio of pseudo bonds of various maturities, similarly to expression (4.6). However Schönbucher (2003) addressed the issue that the default correlation levels that can be reached through this approach are typically too low when compared with empirical default correlations, in the addition to the level of complexity involved in deriving and analysing the resulting dependency structure. In Schönbucher and Schubert (2001), the authors propose an extension of the intensity-based approach to incorporate default correlations using copulas which have been shown to generate realistic time-distribution of the default times. Since the dependency structure is completely described by the copula function, there is liberty in the specification of the copula used in the model which allows for reproduction of various dependency structures between the default times. 


\subsection{Credit Default Swaptions}

Credit default swaptions (hereafter CDS options) are options written on CDS contracts. More precisely, a plain-vanilla CDS option with maturity $T_{m}$ is a European option on a forward credit default swap (hereafter forward CDS). The underlying forward CDS is a CDS contract issued at time $s$ with a start date $T_{m}$ and maturity $T$, with $0 \leq s \leq T_{m}<T$, see Bielecki, Jeanblanc and Rutkowski (2007) for a formal definition. This contract gives default protection over the future interval $\left[T_{m}, T\right]$ but if the reference obligor defaults before the start date, this is $\tau<T_{m}$, the contract is terminated and no payments are made. The value of the forward CDS at time $t \in\left[s, T_{m}\right]$ under the risk-neutral measure is given by

$$
C D S_{f}\left(t, \pi_{f}\right)=\tilde{\mathbb{E}}\left[W_{p r t}^{f}(t)-\pi_{f} \bar{W}_{p r m}^{f}(t) \mid \mathcal{F}_{t}\right]
$$

where, $W_{p r t}^{f}(t)$ is the discounted payoff of the CDS protection leg and $\pi_{f} \bar{W}_{p r m}^{f}(t)=$ $W_{p r m}^{f}(t)$ is the discounted payoff of the CDS premium leg. In addition, we define the forward CDS spread, $\tilde{\pi}_{f}\left(t, T_{m}\right)$, as the variable which will makes the value of the forward CDS contract to be zero at time $t$.

We consider a payer CDS option with a strike rate $K$ and maturity $T_{m}$ on a forward CDS maturing at $T$ and with tenor payment dates $\mathfrak{t}_{1}=T_{m}+\delta, \mathfrak{t}_{2}=T_{m}+2 \delta, \ldots$, $\mathfrak{t}_{N}=T_{m}+N \delta$, with $\delta=\left(T-T_{m}\right) / N$. Upon the option's exercise, which will occur if the reference obligor does not default before $T_{m}$, this is $\tau>T_{m}$, the strike spread $K$ is the fixed rate to be paid (instead of the CDS spread $\tilde{\pi}_{f}\left(t, T_{m}\right)$ ) on the tenor payment dates $\mathfrak{t}_{i}, i=1,2, \ldots, N$, in exchange of the CDS default protection. If the reference obligor defaults before $T_{m}$, the contract will terminate with no payments exchange. Therefore the payoff $V\left(T_{m}\right)$ of the payer CDS options at the option maturity $T_{m}$ is given by

$$
V\left(T_{m}\right)=\mathbb{1}_{\left\{\tau>T_{m}\right\}}\left(C D S_{f}\left(T_{m}, K\right)-C D S_{f}\left(T_{m}, \tilde{\pi}_{f}\left(T_{m}, T_{m}\right)\right)\right)^{+},
$$

where, by definition, $C D S_{f}\left(T_{m}, \tilde{\pi}_{f}\left(T_{m}, T_{m}\right)\right)=0$. For notional convenience we set $\tilde{\pi}_{f}\left(T_{m}, T_{m}\right)=\tilde{\pi}_{f}\left(T_{m}\right)$. As the option will be only exercised if $\tilde{\pi}_{f}\left(T_{m}\right)>K$ by using

\footnotetext{
${ }^{5}$ From the Banc of America Securities, Guide to Credit Default Swaptions, we quote the following: "Credit default swaptions use the lingo payer and receiver, instead of put and call: a payer option is both a put option on credit quality - a bet that credit will deteriorate - and a call option on spreads a bet that spreads will widen".
} 
(4.11), the payoff function can equivalently be written as

$$
\begin{aligned}
V\left(T_{m}\right)= & \mathbb{1}_{\left\{\tau>T_{m}\right\}}\left(\tilde{\mathbb{E}}\left[W_{p r t}^{f}\left(T_{m}\right) \mid \mathcal{F}_{t}\right]-K \tilde{\mathbb{E}}\left[\bar{W}_{p r m}^{f}\left(T_{m}\right) \mid \mathcal{F}_{t}\right]\right)^{+} \\
= & \mathbb{1}_{\left\{\tau>T_{m}\right\}} \mathbb{1}_{\left\{\tilde{\pi}_{f}\left(T_{m}\right)>K\right\}} \tilde{\mathbb{E}}\left[W_{p r t}^{f}\left(T_{m}\right) \mid \mathcal{F}_{t}\right] \\
& -K \mathbb{1}_{\left\{\tau>T_{m}\right\}} \mathbb{1}_{\left\{\tilde{\pi}_{f}\left(T_{m}\right)>K\right\}} \tilde{\mathbb{E}}\left[\bar{W}_{p r m}^{f}\left(T_{m}\right) \mid \mathcal{F}_{t}\right] .
\end{aligned}
$$

Alternatively, by substituting the values $C D S_{f}\left(T_{m}, K\right)$ and $C D S_{f}\left(T_{m}, \tilde{\pi}_{f}\left(T_{m}\right)\right)$ of the forward CDS contracts from (4.11) into the payoff function (4.12), we obtain an expression for the payoff of the payer CDS option in terms of spreads as

$$
V\left(T_{m}\right)=\mathbb{1}_{\left\{\tau>T_{m}\right\}} \tilde{\mathbb{E}}\left[\bar{W}_{p r m}^{f}\left(T_{m}\right) \mid \mathcal{F}_{T_{m}}^{W}\right]\left(\tilde{\pi}_{f}\left(T_{m}\right)-K\right)^{+} .
$$

Then the value of the CDS option, $\mathcal{C}_{\text {swpt }}(t)$, at any time $t \in\left[s, T_{m}\right]$ can be expressed under the risk neutral measure as a discounted payoff, namely,

$$
\mathcal{C}_{\text {swpt }}(t)=\tilde{\mathbb{E}}\left[e^{-\int_{t}^{T_{m}} r(s) d s} V\left(T_{m}\right) \mid \mathcal{F}_{t}\right] .
$$

By using the expression (4.14) for the payoff function, the expectation (4.15) can be reduced to a Black's formula, as proposed by Rutkowski and Armstrong (2009). By an appropriate choice of numeraire that depends on the value of the premium leg and the survival process $\mathbb{Q}\left(t<\tau \mid \mathcal{F}_{t}^{N}\right)$, Rutkowski and Armstrong (2009) define an equivalent probability measure $\overline{\mathcal{Q}}$, and show that the price of the CDS option can be expressed as

$$
\mathcal{C}_{\text {swpt }}(t)=\mathbb{1}_{\{t<\tau\}} \tilde{A}(t) \overline{\mathbb{E}}\left[\left(\tilde{\pi}_{f}\left(t, T_{m}\right)-K\right)^{+} \mid \mathcal{F}_{t}^{W}\right],
$$

where $\overline{\mathbb{E}}$ is the expectation under the $\overline{\mathcal{Q}}$ measure and

$$
\tilde{A}(t)=\mathbb{Q}\left(t<\tau \mid \mathcal{F}_{t}^{W}\right)^{-1} \tilde{\mathbb{E}}\left[\bar{W}_{p r m}(t) \mid \mathcal{F}_{t}^{W}\right] .
$$

In addition, under this new measure, the forward CDS spread is an $\left(\mathcal{F}^{W}, \overline{\mathcal{Q}}\right)$-martingale and its dynamics follow the driftless stochastic differential equation

$$
d \tilde{\pi}_{f}\left(t, T_{m}\right)=\bar{\sigma}(t) \tilde{\pi}_{f}\left(t, T_{m}\right) d \bar{W}(t)
$$

where $\bar{W}$ is a $\overline{\mathcal{Q}}$-Brownian motion. Given that $\tilde{\pi}_{f}(t)$ follows lognormal dynamics and the volatility $\bar{\sigma}$ for different tenor dates is a constant, the value of a credit default swaption with strike $K$ and maturity $T_{m}$ can be calculated by the Black's formula 6

$$
\mathcal{C}_{\text {swpt }}(t)=\mathbb{1}_{\{t<\tau\}} \tilde{A}(t)\left[\tilde{\pi}_{f}\left(t, T_{m}\right) N\left(d_{1}\right)-K N\left(d_{2}\right)\right]
$$

\footnotetext{
${ }^{6}$ It was remarked in Brigo and Morini (2005) that this distributional assumption is inspired by standard models used to model equity and interest rate markets. Jabbour, El-masri and Young (2008) however rejected this hypothesis by showing that the log forward CDS spreads exhibit large positive skewness and excess kurtosis.
} 
where

$$
d_{1}=\frac{\ln \left(\frac{\tilde{\pi}_{f}\left(t, T_{m}\right)}{K}\right)+\frac{\bar{\sigma}^{2}}{2}\left(T_{m}-t\right)}{\bar{\sigma} \sqrt{T_{m}-t}} \quad \text { and } \quad d_{2}=d_{1}-\bar{\sigma} \sqrt{T_{m}-t}
$$

and $\bar{\sigma}$ is the only parameter to be inferred from market data. Although the model is not easily calibrated to quoted data if the market is illiquid, it provides a platform where prices of different options can be translated into implied volatilities thereby giving more information on the market. In addition as noted in Brigo and Morini (2005), the computation of the implied volatilities allows us to assess the implications of different models on the classic strike volatility curve (smile or skew).

\section{Numerical Study}

In this section, we conduct a sensitivity analysis to investigate the impact of the model parameters including correlations and stochastic volatility, on bond prices, credit default swap rates and the value of credit default swaptions.

We assume that the initial term structures of forward rates and forward credit spreads are given by

$$
f(0, T)=0.04-0.04 \sqrt{V(0)} e^{-0.18 T} \quad \text { and } \quad \lambda(0, T)=0.04-0.01 \sqrt{V(0)} e^{-0.1 T}
$$

respectively, with the initial volatility chosen to be $V(0)=1.0$. For simplicity, we specify for $n=1$, the market price of risk $\phi_{j}(t)=\bar{\phi}_{j} \sqrt{V(t)}$ with the scaling factor $\bar{\phi}_{j}=1$ for $j=1,2,3$. In (2.41), we further assume that $\alpha^{V}(t, V)=\kappa^{V}(\bar{V}-V)$ and $\tilde{\sigma}_{1 j}^{V}(t)=z_{1}^{V_{j}} \bar{\sigma}_{1}^{V} \sqrt{V(t)}$ such that

$$
d V(t)=\left[\kappa^{V} \bar{V}-V\left(\kappa^{V}-\sum_{j=1}^{3} z_{1}^{V_{j}} \bar{\sigma}_{1}^{V}\right)\right] d t+\bar{\sigma}_{1}^{V} \sum_{j=1}^{3} z_{1}^{V_{j}} \sqrt{V(t)} d \tilde{W}_{j}(t),
$$

where $z_{1}^{V_{j}}$ is given in (2.22). Table 1 specifies the parameter values used in the analysis that follows.

\begin{tabular}{|c|c|c|c|c|c|c|c|c|c|c|c|}
\hline$a_{01}$ & $a_{11}$ & $b_{01}$ & $b_{11}$ & $\bar{V}$ & $\bar{\sigma}_{1}^{V}$ & $\kappa_{V}$ & $\kappa_{f}$ & $\kappa_{\lambda}$ & $\rho^{V \lambda}$ & $\rho^{V f}$ & $\rho^{f \lambda}$ \\
\hline \hline 0.135 & 0.035 & 0.145 & 0.0045 & 0.7542 & 1.5 & 2.1 & 0.3 & 0.4 & 0.25 & 0.45 & -0.40 \\
\hline
\end{tabular}

Table 1: Parameter values used for simulation studies.

Figure 1 illustrates a possible evolution of the defaultable forward rate surface (3.5) for $n=1$ and $0 \leq t \leq 400$. As it is clear in Figure 1, the proposed hump-shaped level dependent stochastic volatility model can generate a variety of shapes for the defaultable forward rate curve. 


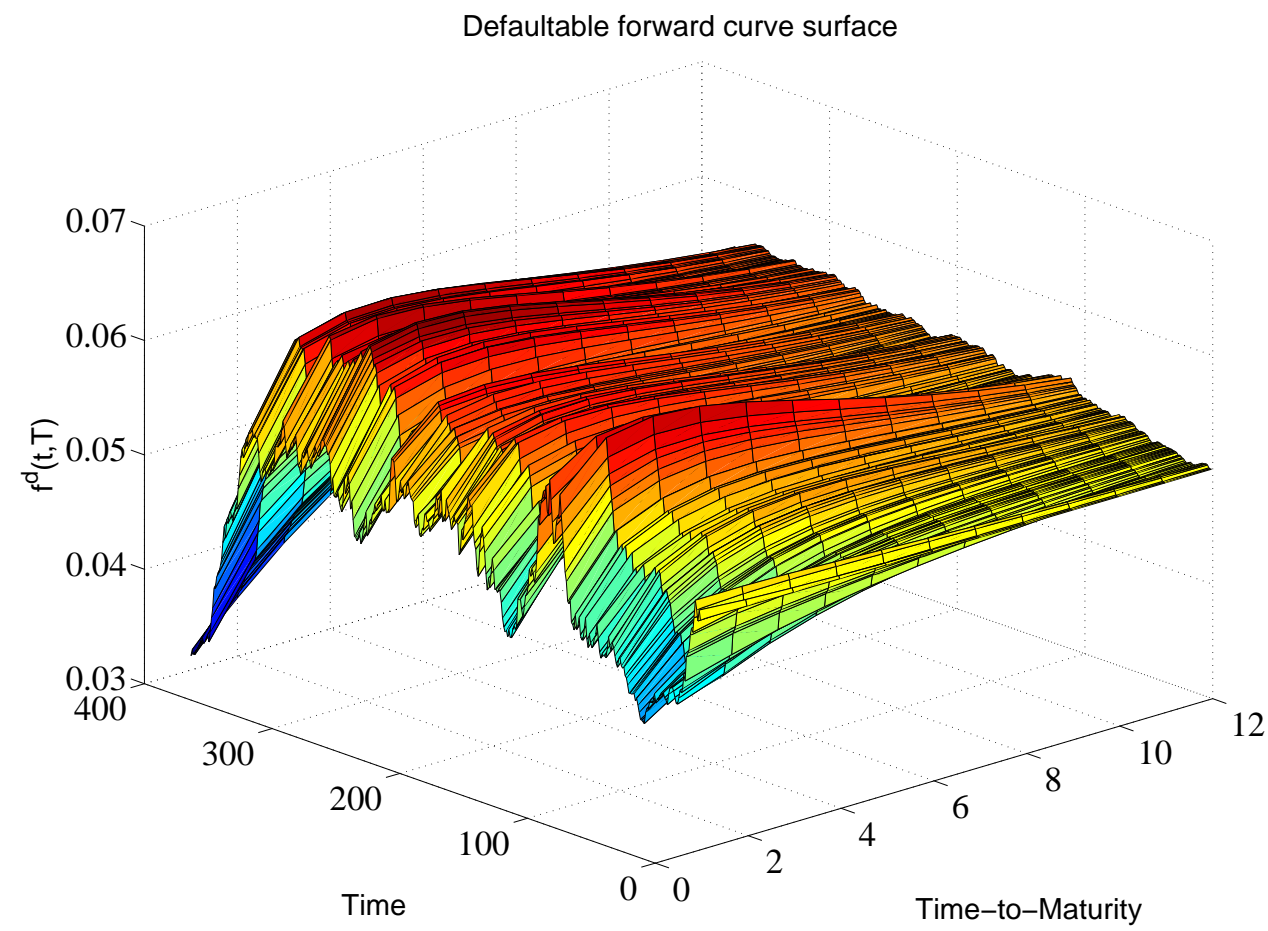

Figure 1: A possible evolution of the defaultable forward rate surface (3.5).

\subsection{Bond Price Distributions}

The pseudo bond price is exponentially affine as presented in (3.11). By simulating the discretised system of the state variables we obtain the simulated distributions of the pseudo bond price with maturity $T=1$.

Figure 2 illustrates the effects of the various correlations, $\rho_{f \lambda}, \rho_{V \lambda}$ and $\rho_{f V}$ together with the parameter value $\bar{\sigma}_{1}^{V}$ on the distribution of the pseudo bond price.

In Figure 2(a) we observe that increasing the correlation $\rho^{\lambda V}$ between the short-term credit spread and the stochastic volatility processes from -0.6 to 0.6 while holding the other correlations constant skews the distribution of the pseudo bond price to the right. This increment is more pronounced when the correlation $\rho^{f V}$ between the short rate and the stochastic volatility process is increased as illustrated in Figure 2(b) since the short rate process, in our case, has higher average volatility than the short term credit spread. In Figure 2(c), we observe that increasing the correlation $\rho^{f \lambda}$ between the short rate and the short term credit spread processes while holding the other correlations constant decreases the kurtosis of the pseudo bond price distribution. Finally, in Figure 2(d) we observe that increasing the parameter $\bar{\sigma}_{1}^{V}$ (which affects the volatility of volatility) 


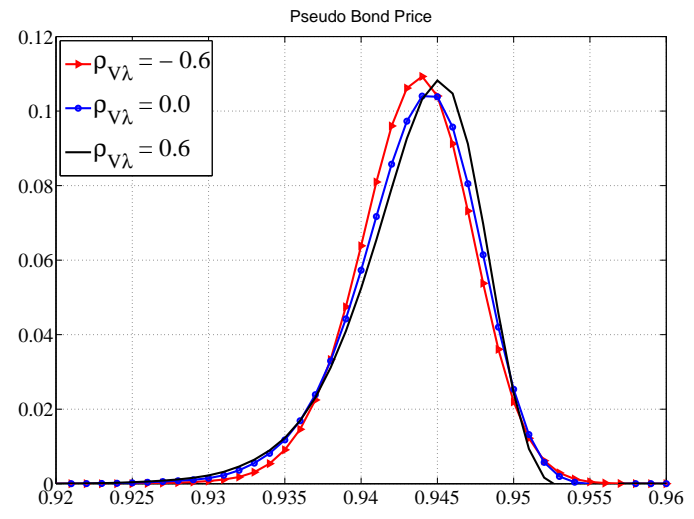

(a)

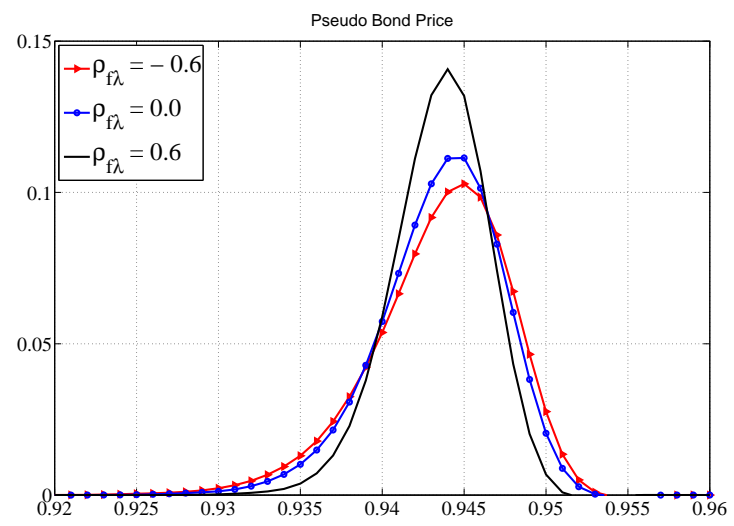

(c)

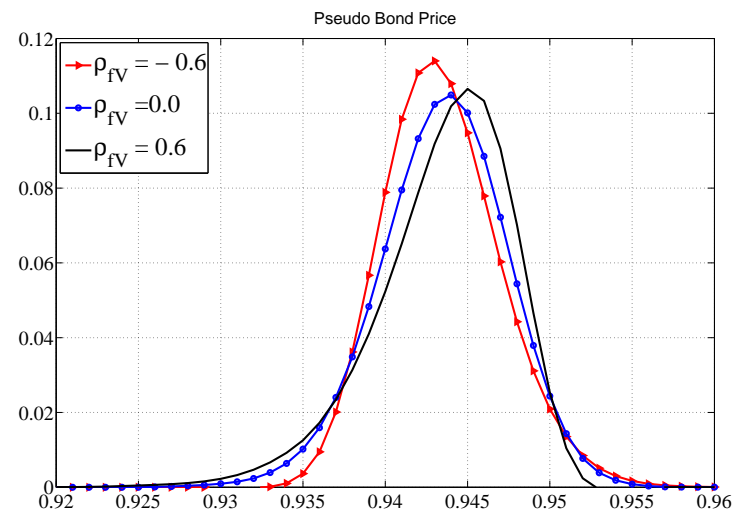

(b)

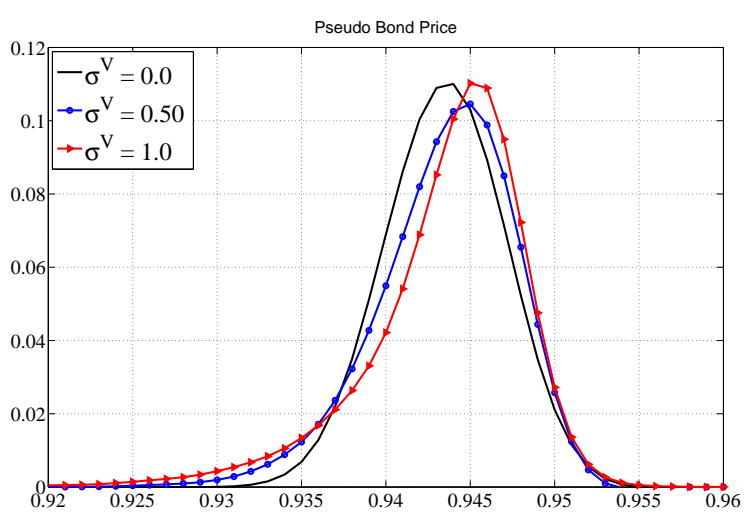

(d)

Figure 2: Panel (a) describes the distribution of the pseudo bond prices under varying $\rho^{V \lambda}$; Panel (b) describes the distribution of the pseudo bond prices under varying $\rho^{V f}$; Panel (c) describes the distribution of the pseudo bond prices under varying $\rho^{f \lambda}$, while Panel (d) describes the distribution of the pseudo bond prices under varying parameter value $\sigma^{V} \equiv \bar{\sigma}_{1}^{V}$ which affects the volatility of volatility. 
skews the distribution of the pseudo bond price to the right, consistent with the empirical evidence noted by D'Souza et al. (2004).

\subsection{Sensitivity Analysis - Credit Default Swaps}

The shape of the credit curves is influenced by the demand and supply for credit protection in the CDS market and reflects the credit quality of the reference entities. By using the parameter values of Table 1, unless otherwise stated, we perform a numerical study to gauge the effect of the correlations and the stochastic volatility to the CDS spreads.

Table 2 shows the effect of increasing maturity on the CDS spread over a 5-year horizon by using (4.6) for varying values of correlation $\rho^{f \lambda}$. We observe that increasing

\begin{tabular}{|c|c|c|c|c|}
\hline Maturity in Years & -0.6 & -0.3 & 0.3 & 0.6 \\
\hline \hline 1 & 243.6273404 & 245.1290045 & 247.1283229 & 248.1230769 \\
\hline 2 & 261.3902994 & 268.311488 & 278.4138084 & 281.302639 \\
\hline 3 & 256.6569148 & 266.2450017 & 284.5637138 & 290.6080929 \\
\hline 4 & 246.1062204 & 260.4087812 & 287.8525595 & 296.1861921 \\
\hline 5 & 232.3321461 & 249.5125039 & 285.0500812 & 297.2019649 \\
\hline
\end{tabular}

Table 2: Simulated CDS Prices (in bps) under varying maturities and correlation $\rho^{f \lambda}$.

the correlation between the market risk and credit risk leads to an increase in the CDS spread. Although Krekel and Wenzel (2006) argued that the effect of this correlation is not significant and need not be taken into consideration when calculating CDS spreads, our results suggest that it has an impact on the CDS premium. A similar conclusion was reached by Berndt et al. (2010). We observe that varying the correlation $\rho^{f \lambda}$ from negative to positive leads to an increase in the CDS spread. Table 3 shows the effect of increasing correlation $\rho^{f V}$ on the CDS spread over a 5-year time horizon. The resulting CDS spread curve exhibits a hump-shaped structure for varying maturities. During nonvolatile market conditions, the cost of protection over a longer term is usually higher as it is difficult to predict cash flows and future events that affect the profitability of a firm over a longer period thereby yielding an increasing CDS curve. 7 From Figure 2(d),

\footnotetext{
${ }^{7}$ There are instances in practice where the higher cost of short-term protection leads to an inverted CDS curve. This implies that a firm faces a greater chance of defaulting within a short term period rather than in the long term. In addition, fears of a sharp rise in the rate of high-yield corporate defaults could prompt investors to seek more short-dated credit protection in a bid to reduce risk.
} 


\begin{tabular}{|c|c|c|c|c|}
\hline Maturity in Years & -0.6 & -0.3 & 0.3 & 0.6 \\
\hline \hline 1 & 247.1131166 & 246.1134583 & 244.6217264 & 244.6267432 \\
\hline 2 & 285.0322943 & 281.8344503 & 269.1525899 & 264.2906356 \\
\hline 3 & 305.7019406 & 300.0149708 & 270.7372222 & 257.9898292 \\
\hline 4 & 321.0644525 & 314.0987507 & 267.8915011 & 247.3428455 \\
\hline 5 & 331.4985844 & 323.3519249 & 261.2202921 & 232.3317749 \\
\hline
\end{tabular}

Table 3: Simulated CDS Prices (in bps) under varying maturities and correlation $\rho^{f V}$.

we observed that increasing the volatility of volatility (through an increase in $\bar{\sigma}_{1}^{V}$ ) leads to an increment in the average pseudo bond prices as it skews the distribution to the right. In Table 4, we observe that this increase in $\bar{\sigma}_{1}^{V}$ leads to a decrease in the CDS spread.

\begin{tabular}{|c|c|c|c|c|}
\hline Maturity in Years & 0.0 & 0.5 & 1.0 & 1.5 \\
\hline \hline 1 & 248.6153846 & 248.1230769 & 247.123254 & 244.6267432 \\
\hline 2 & 287.6505495 & 286.5000878 & 281.8707157 & 265.7167883 \\
\hline 3 & 307.8717201 & 305.9832619 & 295.7395151 & 262.4968975 \\
\hline 4 & 323.5272916 & 320.8278753 & 306.0548877 & 255.1343527 \\
\hline 5 & 334.467374 & 331.2801096 & 312.1298804 & 243.1623909 \\
\hline
\end{tabular}

Table 4: Simulated CDS Prices (in bps) under varying maturities and parameter $\bar{\sigma}_{1}^{V}$.

For a certain recovery assumption, say 40\%, 8 we expect the fair CDS rate to increase as a result of decreasing survival probability leading to widening spreads. Typically, the slope of the CDS spread curve is flatter for higher premium levels and steeper for lower premium levels. Any changes in the shape and perception of the fair premium for credit default swap protection are reflected in the spreads observed in the market. A curve of the survival probability for a reference entity can be inferred from the CDS curve

\footnotetext{
${ }^{8}$ As highlighted in Pan and Singleton (2008), the CDS price under the recovery of market value (RMV) framework is given as a product of the loss given default $L \equiv L G D$ and the default intensity $\tilde{h}(t)$ in the sense that $C D S^{R M V}(t)=g(\tilde{h}(t) L)$ for some function $g$. This implies that the default intensity and the loss given default cannot be identified separately using defaultable bond data alone. Under the recovery of face value (RFV) framework, these two play distinct roles and the CDS pricing relationship is of the form $C D S^{R F V}(t)=L f(\tilde{h}(t))$. This has the immediate consequence that under $R F V$, the explicit dependence of $C D S(t)$ on $L G D$ implies that the ratio of two CDS spreads on contracts of different maturities does not depend of $L G D$ but contains information about $\tilde{h}(t)$. We adopt this model of recovery in our formulation.
} 
and can be seen to be a decreasing function to maturity. Higher recovery rates implied by different ratings classes give rise to lower the CDS spread for bonds with 5-year maturities.

\subsection{Sensitivity Analysis - Credit Default Swaptions}

To illustrate the model's properties we compute the swaption prices based on (4.19) for varying parameter values. At time $t=0$, we calculate the price of a swaption with maturity $T_{m}=0.5$ issued on a credit default swap that has a defaultable bond (based on the framework developed in Section $[3$ ) with a maturity of $T=2$ years as its underlying and the default protection is required for the period $[0.5,2.5]$. In addition, we use the following parameters for the calculation of the swaption prices: recovery rate $\mathcal{R}=40 \%$, volatility of the forward CDS rate $\bar{\sigma}=0.49$ with $N=400$.

Figure 3 gives the credit default swaption price under varying strike rates and correlation $\rho^{f \lambda}$ between short rate $r(t)$ and short term credit spread $10 c(t)$. We observe that increasing $K$ leads to a decrease in the credit default swaption rate which is in line with the market behavior that deep in-the-money options trade higher than at-the-money and out-of-the-money options. For deep in-the-money options, negative correlation $\rho^{f \lambda}$ is seen to produce higher swaption prices. We observe that $\rho^{f \lambda}<0$ implies that when default risk is high (hence higher probability of default by the obligor), interest rates are low and the value of CDS contract is high, leading to higher swaption prices. Conversely for $\rho^{f \lambda}>0$ when the default risk is high, the interest rates are also high giving rise to lower CDS prices and therefore lower swaption prices.

From Figure 4, we observe that negative correlation $\rho^{f V}$ produces higher swaption prices for deep in-the-money options as compared to positive correlation. In Figure 5 , the value of the credit default swaption price is given as a function of the parameter value $\bar{\sigma}_{1}^{V}$ which affects the volatility of volatility, as given in (5.1). We observe that increasing $\bar{\sigma}_{1}^{V}$ leads to a decrease in the swaption price, with the effect being more for deep in the money options. This follows from the observation made in Table 4 that an increase in $\bar{\sigma}_{1}^{V}$ leads to a reduction in the CDS rates.

Figure 6 investigates the time sensitivity of the swaption and we observe that the credit swaption becomes more sensitive to changes in the volatility assumption the longer

\footnotetext{
${ }^{9}$ It was noted in Schönbucher (2004) that the value of the volatility $\tilde{\pi}_{f}=0.4$ is of an acceptable level for simulation purposes.

${ }^{10}$ We recall that in this case, the short term credit spread coincides with the default intensity given that we are considering the pre-default bond price.
} 


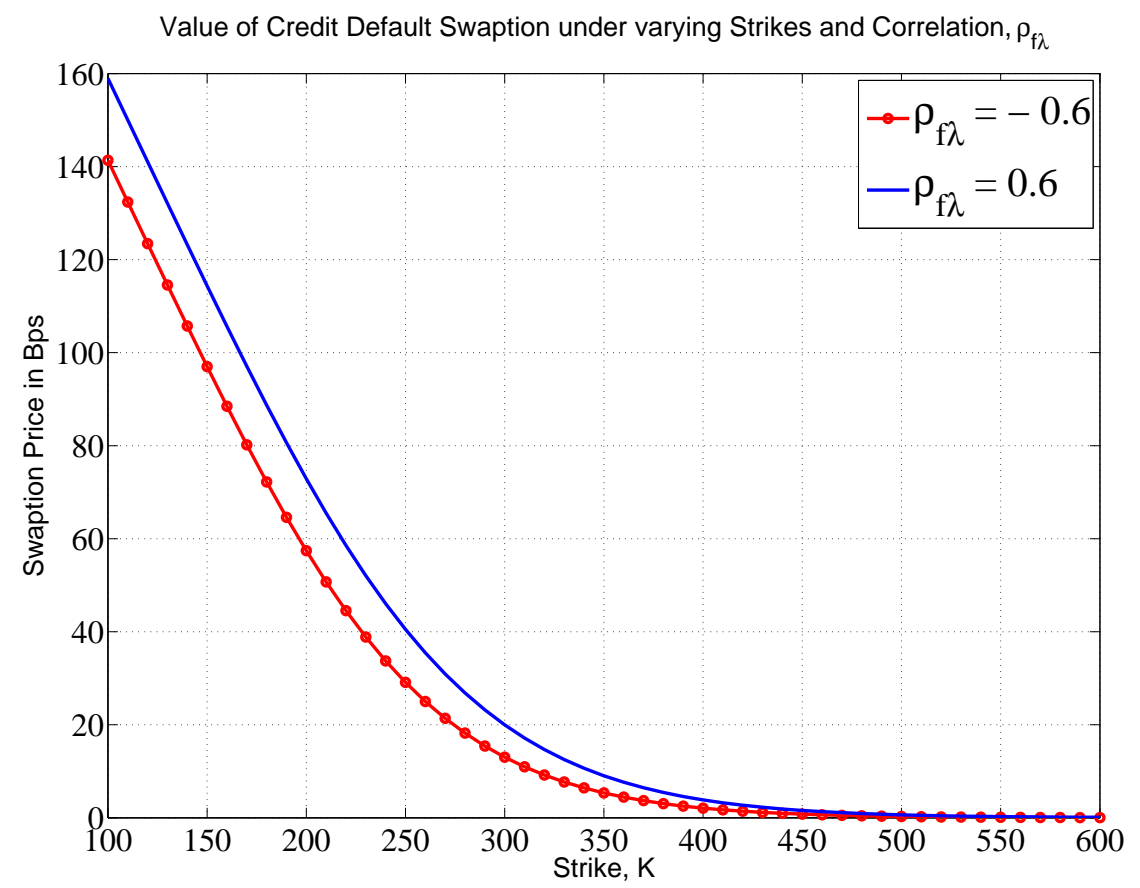

Figure 3: Credit default swaption price given by equation (4.19) under varying strikes and correlation $\rho_{f \lambda}$ between interest rate $r(t)$ and short term credit spread $c(t)$.

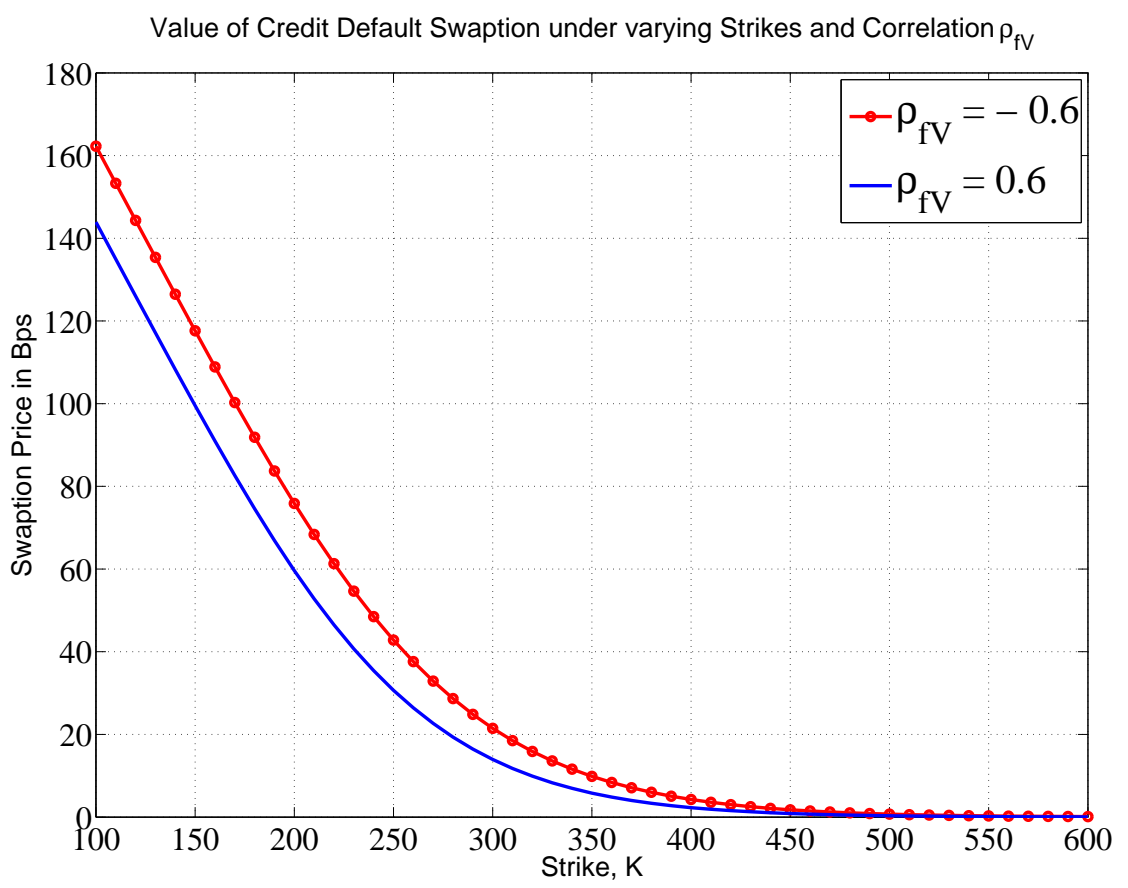

Figure 4: Credit default swaption price given by equation (4.19) under varying strikes and correlation $\rho_{f V}$ between interest rate $r(t)$ and the stochastic volatility process $V(t)$. 


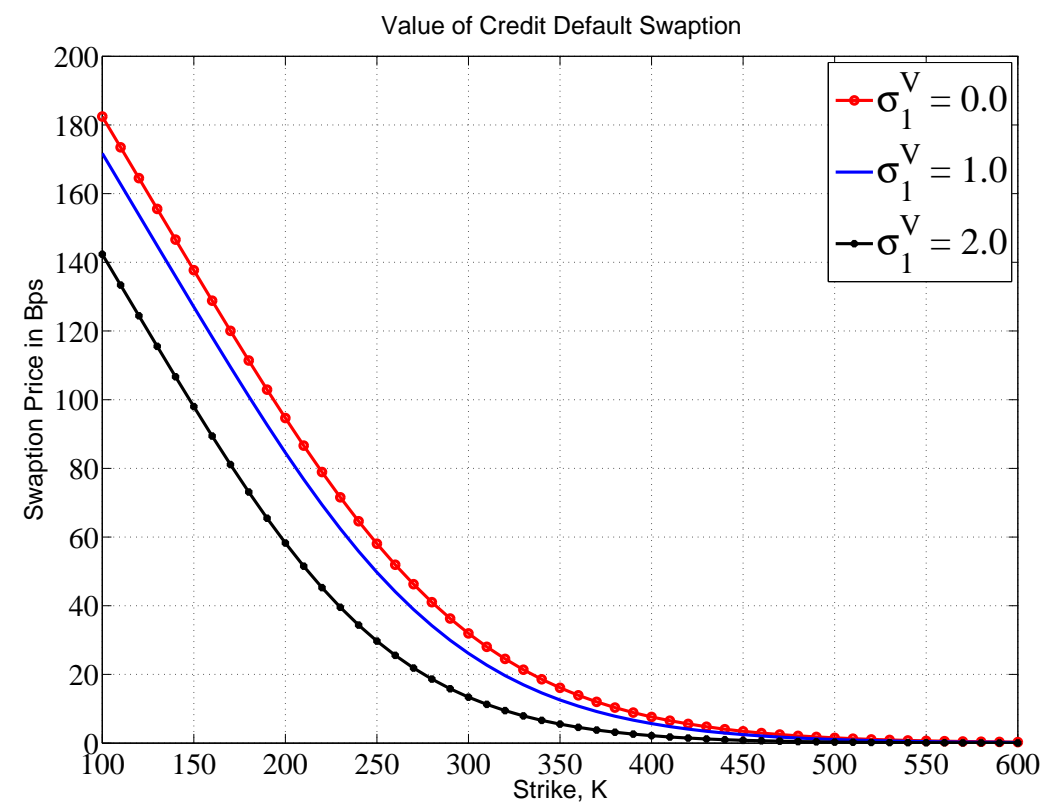

Figure 5: Credit Default Swaption prices under varying strikes and volatility of volatility, where we have denoted the volatility of volatility by $\sigma_{1}^{V}$.

the time to maturity. It is observed that an increase in the volatility of the forward CDS rate $\bar{\sigma}$ leads to an increase in the swaption price.

\section{Conclusion}

This paper presents a Markovian HJM model for the defaultable term structure with unspanned stochastic volatility. Even though, the volatility of the default-free and defaultable term structure has been restricted so as to allow finite dimensional realisations of the state space, it is nevertheless level dependent, hump-shaped and depends on the general unspanned stochastic volatility process. A tractable formula for the default-free and the defaultable zero coupon bond prices are derived, expressing them as an exponential affine function of state variables.

We also demonstrated how the framework can be applied to price credit default swaps and swaptions and we derived formulas that approximate single-name CDS prices and show how this could be extended to include counterparty risk. From the numerical study, we observe that the model captures the stylized features of credit default swaps and swaptions subject to the assumptions on the recovery of the defaultable bond as well as the time to maturity of the option. 


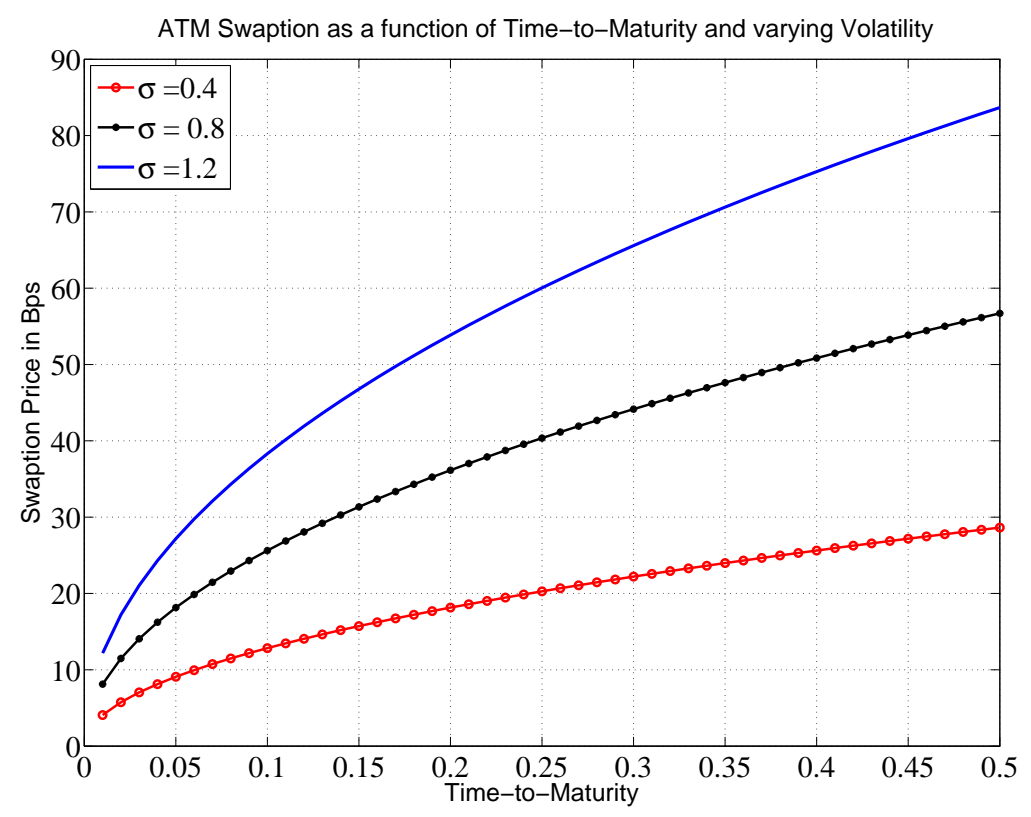

Figure 6: The value of an ATM swaption for varying time to maturity of the option and different volatility values for the CDS price dynamics. We note that we have denoted the volatility of CDS spread by $\sigma$. That is, $\bar{\sigma}=\sigma$.

The model offers a general yet tractable framework to analyse and measure the extent to which volatility can be spanned in the credit risk market. To our knowledge there is no empirical study on this very important feature of credit risk volatility. This paper provides a theoretical framework based on a state space representation that could be adapted to the study and estimation of stochastic volatility in defaultable markets. This task we leave to future research.

\section{Appendix}

\section{A Proof of Proposition 2.45 on Defaultable Bond Price}

We note that the defaultable bond price under the expectation is given by

$$
P^{d}(t, T)=\tilde{\mathbb{E}}\left[e^{-\int_{t}^{T} r(s) d s} \mathbb{1}_{\{\tau>T\}} \mid \mathcal{F}_{t}\right] .
$$

In addition, it was also shown in Lando (1998) that

$$
\tilde{\mathbb{E}}\left[\mathbb{1}_{\{\tau \geq T\}} \mid \mathcal{F}_{t}^{W} \vee \mathcal{F}_{t}^{\tau}\right]=\mathbb{1}_{\{\tau>T\}} e^{-\int_{t}^{T} \tilde{h}(s) d s} .
$$


It then follows that the RHS of (A.1) can be written as

$$
\begin{aligned}
\tilde{\mathbb{E}}\left[e^{-\int_{t}^{T} r(s) d s}\right. & \left.\mathbb{1}_{\{\tau>T\}} \mid \mathcal{F}_{t}\right]=\tilde{\mathbb{E}}\left[\tilde{\mathbb{E}}\left(e^{-\int_{t}^{T} r(s) d s} \mathbb{1}_{\{\tau>T\}} \mid \mathcal{F}_{t}^{W} \vee \mathcal{F}_{t}^{\tau}\right) \mid \mathcal{F}_{t}\right] \\
& =\tilde{\mathbb{E}}\left[e^{-\int_{t}^{T} r(s) d s} \tilde{\mathbb{E}}\left(\mathbb{1}_{\{\tau>T\}} \mid \mathcal{F}_{t}^{W} \vee \mathcal{F}_{t}^{\tau}\right) \mid \mathcal{F}_{t}\right] \\
& =\mathbb{1}_{\{\tau>T\}} \tilde{\mathbb{E}}\left[e^{-\int_{t}^{T}(r(s)+\tilde{h}(s)) d s} \mid \mathcal{F}_{t}^{W}\right] .
\end{aligned}
$$

Hence the proof of Proposition 2.45,

\section{B Proof of Proposition 3.1 on the Defaultable For- ward Rate}

We recall from (2.38) that under the risk-neutral measure, the dynamics of the default-free forward rate can be written as

$f(t, T)=f(0, T)+\sum_{i=1}^{n} \int_{0}^{t} \tilde{\sigma}_{i 1}^{f}\left(u, T, V_{i}\right) \int_{u}^{T} \tilde{\sigma}_{i 1}^{f}\left(u, s, V_{i}\right) d s d u+\sum_{i=1}^{n} \int_{0}^{t} \tilde{\sigma}_{i 1}^{f}\left(u, T, V_{i}\right) d \tilde{W}_{i}(u)$.

Using the volatility specifications (3.1), the stochastic integral equation (B.1) becomes

$$
\begin{aligned}
f(t, T)=f(0, T) & +\sum_{i=1}^{n} \int_{0}^{t} r(u) V_{i}(u)\left[a_{0 i}+a_{1 i}(T-u)\right] e^{-\kappa_{i}^{f}(T-u)} \int_{u}^{T}\left[a_{0 i}+a_{1 i}(s-u)\right] e^{-\kappa_{i}^{f}(s-u)} d s d u \\
& +\sum_{i=1}^{n} \int_{0}^{t} \sqrt{r(u) V_{i}(u)}\left[a_{0 i}+a_{1 i}(T-u)\right] e^{-\kappa_{i}^{f}(T-u)} d \tilde{W}_{i}(u) .
\end{aligned}
$$

By using the property $T-u=(T-t)+(t-u)$, and perform standard algebraic manipulations, equation (B.2) becomes

$$
\begin{aligned}
f(t, T) & =f(0, T)+\sum_{i=1}^{n} \frac{a_{1 i}}{\kappa_{i}^{f}}\left(\frac{1}{\kappa_{i}^{f}}+\frac{a_{0 i}}{a_{1 i}}\right)\left[a_{0 i}+a_{1 i}(T-t)\right] e^{-\kappa_{i}^{f}(T-t)} \Phi_{2 i}(t) \\
& +\sum_{i=1}^{n}\left[\frac{a_{1 i} a_{1 i}}{\kappa_{i}^{f}}\left(\frac{1}{\kappa_{i}^{f}}+\frac{a_{0 i}}{a_{1 i}}\right)+\frac{a_{1 i}}{\kappa_{i}^{f}}\left(\frac{a_{1 i}}{\kappa_{i}^{f}}+2 a_{0 i}\right)(T-t)+\frac{a_{1 i}}{\kappa_{i}^{f}}(T-t)^{2}\right] e^{-\kappa_{i}^{f}(T-t)} \Phi_{3 i}(t) \\
& +\sum_{i=1}^{n} \frac{\left(a_{1 i}\right)^{2}}{\kappa_{i}^{f}}\left(\frac{1}{\kappa_{i}^{f}}+\frac{a_{0 i}}{a_{1 i}}\right) e^{-\kappa_{i}^{f}(T-t)} \Phi_{4 i}(t) \\
& -\sum_{i=1}^{n} \frac{a_{1 i}}{\kappa_{i}^{f}}\left[\frac{a_{1 i}}{\kappa_{i}^{f}}+2 a_{0 i}+2 a_{1 i}(T-t)\right] e^{-2 \kappa_{i}^{f}(T-t)} \Phi_{5 i}(t)-\sum_{i=1}^{n} \frac{\left(a_{1 i}\right)^{2}}{\kappa_{i}^{f}} e^{-2 \kappa_{i}^{f}(T-t)} \Phi_{6 i}(t) \\
& +\sum_{i=1}^{n} z_{i}^{f_{1}}\left[a_{0 i}+a_{1 i}(T-t)\right] e^{-\kappa_{i}^{f}(T-t)} x_{1 i}(t)+\sum_{i=1}^{n} z_{i}^{f_{1}} a_{1 i} e^{-\kappa_{i}^{f}(T-t)} \Phi_{1 i}(t),
\end{aligned}
$$


where

$$
\left\{\begin{array}{l}
x_{1 i}(t)=\int_{0}^{t} \sqrt{r(u) V_{i}(u)} e^{-\kappa_{i}^{f}(t-u)} d \tilde{W}_{i}(u), \\
\Phi_{1 i}(t)=\int_{0}^{t} \sqrt{r(u) V_{i}(u)}(t-u) e^{-\kappa_{i}^{f}(t-u)} d \tilde{W}_{i}(u), \\
\Phi_{2 i}(t)=\int_{0}^{t} r(u) V_{i}(u) e^{-\kappa_{i}^{f}(t-u)} d u, \\
\Phi_{3 i}(t)=\int_{0}^{t} r(u) V_{i}(u) e^{-2 \kappa_{i}^{f}(t-u)} d u, \\
\Phi_{4 i}(t)=\int_{0}^{t} r(u) V_{i}(u)(t-u) e^{-\kappa_{i}^{f}(t-u)} d u, \\
\Phi_{5 i}(t)=\int_{0}^{t} r(u) V_{i}(u)(t-u) e^{-2 \kappa_{i}^{f}(t-u)} d u, \\
\Phi_{6 i}(t)=\int_{0}^{t} r(u) V_{i}(u)(t-u)^{2} e^{-2 \kappa_{i}^{f}(t-u)} d u,
\end{array}\right.
$$

Thus, the forward rate process is Markovian and more specifically is affine in the state space variables since

$$
f(t, T)=f(0, T)+\sum_{i=1}^{n} B_{x_{1 i}}(T-t) x_{1 i}(t)+\sum_{i=1}^{n} \sum_{j=1}^{6} B_{\Phi_{j i}}(T-t) \Phi_{j i}(t),
$$

where $B_{x_{1 i}}(T-t)$ and $B_{\Phi_{j i}}(T-t)$ are given in (3.6) $)$.

Similarly, we recall from (2.39) that the forward credit spread dynamics under the risk-neutral measure can be written as

$$
\begin{aligned}
\lambda(t, T) & =\sum_{i=1}^{2 n} \int_{0}^{t} \tilde{\sigma}_{i}^{\lambda}(u, T) \int_{u}^{T} \tilde{\sigma}_{i}^{\lambda}(u, s) d s d u+\sum_{i=1}^{2 n} \int_{0}^{t}\left(\tilde{\sigma}_{i}^{\lambda}(u, T) \int_{u}^{T} \tilde{\sigma}_{i}^{f}(u, s) d s+\tilde{\sigma}_{i}^{f}(u, T) \int_{u}^{T} \tilde{\sigma}_{i}^{\lambda}(u, s) d s\right) d u \\
& +\sum_{i=1}^{2 n} \int_{0}^{t} \tilde{\sigma}_{i}^{\lambda}(u, T) d \tilde{W}_{i}(u) .
\end{aligned}
$$

Then by using the volatility specifications of Assumption 3.1, the drift components in the forward credit spread representation (B.6) are given by (recall that $z_{i}^{f_{1}}=1$ so that $\left.z_{i}^{\lambda_{1}} z_{i}^{f_{1}}=z_{i}^{\lambda_{1}}\right)$

$$
\begin{aligned}
& \sum_{i=1}^{2 n} \int_{0}^{t} \tilde{\sigma}_{i}^{\lambda}(u, T) \int_{u}^{T} \tilde{\sigma}_{i}^{f}(u, s) d s d u \\
& \quad=\sum_{i=1}^{n} z_{i}^{\lambda_{1}}\left\{\left[\frac{a_{0 i} b_{0 i}}{\kappa_{i}^{f}}+\frac{a_{1 i} b_{0 i}}{\left(\kappa_{i}^{f}\right)^{2}}+\left(\frac{a_{0 i} b_{1 i}}{\kappa_{i}^{f}}+\frac{a_{1 i} b_{1 i}}{\left(\kappa_{i}^{f}\right)^{2}}\right)(T-t)\right] e^{-\kappa_{i}^{\lambda}(T-t)} \Phi_{7 i}(t)\right. \\
& \quad-\left[\frac{a_{0 i} b_{0 i}}{\kappa_{i}^{f}}+\frac{a_{1 i} b_{0 i}}{\left(\kappa_{i}^{f}\right)^{2}}+\left(\frac{a_{1 i} b_{0 i}}{\kappa_{i}^{f}}+\frac{a_{0 i} b_{1 i}}{\kappa_{i}^{f}}+\frac{a_{1 i} b_{1 i}}{\left(\kappa_{i}^{f}\right)^{2}}\right)(T-t)+\frac{a_{1 i} b_{1 i}}{\kappa_{i}^{f}}(T-t)^{2}\right] e^{-\left(\kappa_{i}^{f}+\kappa_{i}^{\lambda}\right)(T-t)} \Phi_{8 i}(t) \\
& \quad+\left(\frac{a_{0 i} b_{0 i}}{\kappa_{i}^{f}}+\frac{a_{1 i} b_{1 i}}{\left(\kappa_{i}^{f}\right)^{2}}\right) e^{-\kappa_{i}^{\lambda}(T-t)} \Phi_{9 i}(t) \\
& \quad-\left[\frac{a_{1 i} b_{0 i}}{\kappa_{i}^{f}}+\frac{a_{0 i} b_{1 i}}{\kappa_{i}^{f}}+\frac{a_{1 i} b_{1 i}}{\left(\kappa_{i}^{f}\right)^{2}}-2 \frac{a_{1 i} b_{1 i}}{\kappa_{i}^{f}}(T-t)\right] e^{-\left(\kappa_{i}^{f}+\kappa_{i}^{\lambda}\right)(T-t)} \Phi_{10 i}(t) \\
& \left.\left.\quad-\frac{a_{1 i} b_{1 i}}{\kappa_{i}^{f}} e^{-\left(\kappa_{i}^{f}+\kappa_{i}^{\lambda}\right)(T-t)} \Phi_{11 i}(t)\right)\right\}
\end{aligned}
$$


and

$$
\begin{aligned}
& \sum_{i=1}^{2 n} \int_{0}^{t} \tilde{\sigma}_{i}^{f}(u, T) \int_{u}^{T} \tilde{\sigma}_{i}^{\lambda}(u, s) d s d u=\sum_{i=1}^{n} z_{i}^{\lambda_{1}}\{ \\
& \quad-\left[\frac{a_{0 i} b_{0 i}}{\kappa_{i}^{\lambda}}+\frac{a_{0 i} b_{1 i}}{\left(\kappa_{i}^{\lambda}\right)^{2}}+\left(\frac{a_{0 i} b_{1 i}}{\kappa_{i}^{\lambda}}+\frac{a_{1 i} b_{0 i}}{\kappa_{i}^{\lambda}}+\frac{a_{1 i} b_{1 i}}{\left(\kappa_{i}^{\lambda}\right)^{2}}\right)(T-t)+\frac{a_{1 i} b_{1 i}}{\kappa_{i}^{\lambda}}(T-t)^{2}\right] e^{-\left(\kappa_{i}^{f}+\kappa_{i}^{\lambda}\right)(T-t)} \Phi_{8 i}(t) \\
& \quad-\left[\frac{b_{1 i} a_{0 i}}{\kappa_{i}^{\lambda}}+\frac{b_{0 i} a_{1 i}}{\kappa_{i}^{\lambda}}+\frac{a_{1 i} b_{1 i}}{\left(\kappa_{i}^{\lambda}\right)^{2}}-2 \frac{a_{1 i} b_{1 i}}{\kappa_{i}^{\lambda}}(T-t)\right] e^{-\left(\kappa_{i}^{f}+\kappa_{i}^{\lambda}\right)(T-t)} \Phi_{10 i}(t) \\
& \quad-\frac{a_{1 i} b_{1 i}}{\kappa_{i}^{\lambda}} e^{-\left(\kappa_{i}^{f}+\kappa_{i}^{\lambda}\right)(T-t)} \Phi_{11 i}(t)+\left[\frac{a_{0 i} b_{0 i}}{\kappa_{i}^{\lambda}}+\frac{a_{0 i} b_{1 i}}{\left(\kappa_{i}^{\lambda}\right)^{2}}+\left(\frac{a_{1 i} b_{0 i}}{\kappa_{i}^{\lambda}}+\frac{a_{1 i} b_{1 i}}{\left(\kappa_{i}^{\lambda}\right)^{2}}\right)(T-t)\right] e^{-\kappa_{i}^{f}(T-t)} \Phi_{12 i}(t) \\
& \left.+\left(\frac{a_{0 i} b_{0 i}}{\kappa_{i}^{\lambda}}+\frac{a_{1 i} b_{1 i}}{\left(\kappa_{i}^{\lambda}\right)^{2}}\right) e^{-\kappa_{i}^{f}(T-t)} \Phi_{13 i}(t)\right\},
\end{aligned}
$$

and finally

$$
\begin{aligned}
& \sum_{i=1}^{2 n} \int_{0}^{t} \tilde{\sigma}_{i}^{\lambda}(u, T) \int_{u}^{T} \tilde{\sigma}_{i}^{\lambda}(u, s) d s d u=\sum_{i=1}^{n}\left\{\frac{b_{1 i}}{\kappa_{i}^{\lambda}}\left(\frac{1}{\kappa_{i}^{\lambda}}+\frac{b_{0 i}}{b_{1 i}}\right)\left[b_{0 i}+b_{1 i}(T-t)\right] e^{-\kappa_{i}^{\lambda}(T-t)} \Phi_{14 i}(t)\right. \\
& \quad+\left[\frac{b_{1 i} b_{1 i}}{\kappa_{i}^{\lambda}}\left(\frac{1}{\kappa_{i}^{\lambda}}+\frac{b_{0 i}}{b_{1 i}}\right)+\frac{b_{1 i}}{\kappa_{i}^{\lambda}}\left(\frac{b_{1 i}}{\kappa_{i}^{\lambda}}+2 b_{0 i}\right)(T-t)+\frac{b_{1 i}}{\kappa_{i}^{\lambda}}(T-t)^{2}\right] e^{-\kappa_{i}^{\lambda}(T-t)} \Phi_{15 i}(t) \\
& \quad+\frac{\left(b_{1 i}\right)^{2}}{\kappa_{i}^{\lambda}}\left(\frac{1}{\kappa_{i}^{\lambda}}+\frac{b_{0 i}}{b_{1 i}}\right) e^{-\kappa_{i}^{\lambda}(T-t)} \Phi_{16 i}(t) \\
& \left.\quad-\frac{b_{1 i}}{\kappa_{i}^{\lambda}}\left[\frac{b_{1 i}}{\kappa_{i}^{\lambda}}+2 b_{0 i}+2 b_{1 i}(T-t)\right] e^{-2 \kappa_{i}^{\lambda}(T-t)} \Phi_{17 i}(t)-\frac{\left(b_{1 i}\right)^{2}}{\kappa_{i}^{\lambda}} e^{-2 \kappa_{i}^{\lambda}(T-t)} \Phi_{18 i}(t)\right\}
\end{aligned}
$$

where we introduce the additional state variables

$$
\left\{\begin{array}{l}
\Phi_{7 i}(t)=\int_{0}^{t} \eta_{i}(u) e^{-\kappa_{i}^{\lambda}(t-u)} d u, \quad \Phi_{8 i}(t)=\int_{0}^{t} \eta_{i}(u) e^{-\left(\kappa_{i}^{f}+\kappa_{i}^{\lambda}\right)(t-u)} d u, \\
\Phi_{9 i}(t)=\int_{0}^{t} \eta_{i}(u)(t-u) e^{-\kappa_{i}^{\lambda}(t-u)} d u, \quad \Phi_{10 i}(t)=\int_{0}^{t} \eta_{i}(u)(t-u) e^{-\left(\kappa_{i}^{f}+\kappa_{i}^{\lambda}\right)(t-u)} d u \\
\Phi_{11 i}(t)=\int_{0}^{t} \eta_{i}(u)(t-u)^{2} e^{-\left(\kappa_{i}^{f}+\kappa_{i}^{\lambda}\right)(t-u)} d u, \quad \Phi_{12 i}(t)=\int_{0}^{t} \eta_{i}(u) e^{-\kappa_{i}^{f}(t-u)} d u \\
\Phi_{13 i}(t)=\int_{0}^{t} \eta_{i}(u)(t-u) e^{-\kappa_{i}^{f}(t-u)} d u, \quad \Phi_{14 i}(t)=\int_{0}^{t} c(u) V_{i}(u) e^{-\kappa_{i}^{\lambda}(t-u)} d u \\
\Phi_{15 i}(t)=\int_{0}^{t} c(u) V_{i}(u) e^{-2 \kappa_{i}^{\lambda}(t-u)} d u, \quad \Phi_{16 i}(t)=\int_{0}^{t} c(u) V_{i}(u)(t-u) e^{-\kappa_{i}^{\lambda}(t-u)} d u \\
\Phi_{17 i}(t)=\int_{0}^{t} c(u) V_{i}(u)(t-u) e^{-2 \kappa_{i}^{\lambda}(t-u)} d u, \quad \Phi_{18 i}(t)=\int_{0}^{t} c(u) V_{i}(u)(t-u)^{2} e^{-2 \kappa_{i}^{\lambda}(t-u)} d u
\end{array}\right.
$$

where $\eta_{i}(t)=V_{i}(t) \sqrt{r(t) c(t)}$. We note in addition that,

$$
\begin{gathered}
\sum_{i=1}^{2 n} \int_{0}^{t} \tilde{\sigma}_{i}^{\lambda}(u, T) d \tilde{W}_{i}(u)=\sum_{i=1}^{n} z_{i}^{\lambda_{1}}\left[b_{0 i}+b_{1 i}(T-t)\right] e^{-\kappa_{i}^{\lambda}(T-t)} x_{2 i}(t) \\
+\sum_{i=1}^{n} z_{i}^{\lambda_{2}}\left[b_{0 i}+b_{1 i}(T-t)\right] e^{-\kappa_{i}^{\lambda}(T-t)} x_{3 i}(t)+\sum_{i=1}^{n} z_{i}^{\lambda_{1}} b_{1 i} e^{-\kappa_{i}^{\lambda}(T-t)} \Phi_{19 i}(t) \\
\quad+\sum_{i=1}^{n} z_{i}^{\lambda_{2}} b_{1 i} e^{-\kappa_{i}^{\lambda}(T-t)} \Phi_{20 i}(t)
\end{gathered}
$$


where we introduce the state variables

$$
\left\{\begin{array}{l}
x_{2 i}(t)=\int_{0}^{t} \sqrt{c(u) V_{i}(u)} e^{-\kappa_{i}^{\lambda}(t-u)} d \tilde{W}_{i}(u), \quad x_{3 i}(t)=\int_{0}^{t} \sqrt{c(u) V_{i}(u)} e^{-\kappa_{i}^{\lambda}(t-u)} d \tilde{W}_{n+i}(u), \\
\Phi_{19 i}(t)=\int_{0}^{t} c(u) V_{i}(u)(t-u) e^{-\kappa_{i}^{\lambda}(t-u)} d \tilde{W}_{i}(u), \quad \Phi_{20 i}(t)=\int_{0}^{t} c(u) V_{i}(u)(t-u) e^{-\kappa_{i}^{\lambda}(t-u)} d \tilde{W}_{n+i}(u) .
\end{array}\right.
$$

Consequently, the forward credit spread satisfies

$$
\lambda(t, T)=\lambda(0, T)+\sum_{i=1}^{n} \sum_{j=2}^{3} B_{x_{j i}}(T-t) x_{j i}(t)+\sum_{i=1}^{n} \sum_{j=7}^{20} B_{\Phi_{j i}}(T-t) \Phi_{j i}(t),
$$

where the coefficients $B_{x_{j i}}(T-t)$ and $B_{\Phi_{j i}}(T-t)$ are specified in (3.7) and (3.8). It follows from the definition $f^{d}(t, T)=f(t, T)+\lambda(t, T)$ and (B.5) and (B.13) that the defaultable forward rate admits finite dimensional affine realisation

$$
f^{d}(t, T)=f^{d}(0, T)+\sum_{i=1}^{n} \sum_{j=1}^{3} B_{x_{j i}}(T-t) x_{j i}(t)+\sum_{i=1}^{n} \sum_{j=1}^{20} B_{\Phi_{j i}}(T-t) \Phi_{j i}(t) .
$$

Hence the proof of Proposition 3.1.

\section{Proof of Proposition 3.4 for the Exponential Affine Bond Price formula}

Straightforward application of (B.14) for the affine Markovian forward rate into the definition of the defaultable bond price formula, $\bar{P}^{d}(t, T)=\exp \left(-\int_{t}^{T} f^{d}(t, s) d s\right)$ yields $\bar{P}^{d}(t, T)=\frac{\bar{P}^{d}(0, T)}{\bar{P}^{d}(0, t)} \exp \left(-\sum_{i=1}^{n} \sum_{j=1}^{3} x_{j i}(t) \int_{t}^{T} B_{x_{j i}}(s-t) d s-\sum_{i=1}^{n} \sum_{j=1}^{20} \Phi_{j i}(t) \int_{t}^{T} B_{\Phi_{j i}}(s-t) d s\right)$.

We proceed to integrate the deterministic functions $B_{x_{j i}}$ and $B_{\Phi_{j i}}$ (See Proposition 3.1) in the exponent with respect to maturity. Substituting equations (3.12) - (3.15) into the general exponential defaultable bond price expression (C.1) yields (3.11) in Proposition 3.4 .

Hence the proof of Proposition 3.4 . 


\section{Proof of Proposition 4.1 for Standard CDS}

Given that the CDS spread $\tilde{\pi}(t)$ is the value $\pi$ that makes the value of the swap contract to be zero, from (4.3) and (4.2) it follows that

$$
\tilde{\pi}(t)=\frac{(1-\mathcal{R}) \tilde{\mathbb{E}}\left[e^{-\int_{t}^{\tau} r(s) d s} \mathbb{1}_{\{t<\tau \leq T\}} \mid \mathcal{F}_{t}\right]}{\sum_{i=1}^{N} \delta_{i} \tilde{\mathbb{E}}\left[e^{-\int_{t}^{t_{i}} r(s) d s} \mathbb{1}_{\left\{\tau>t_{i}\right\}} \mid \mathcal{F}_{t}\right]+\tilde{\mathbb{E}}\left[\left(\tau-t_{\tau-1}\right) e^{-\int_{t}^{\tau} r(s) d s} \mathbb{1}_{\{t<\tau<T\}} \mid \mathcal{F}_{t}\right]} .
$$

Note that $\mathbb{1}_{\{\tau>T\}}=\mathbb{1}_{\{\tau>t\}} \mathbb{1}_{\{\tau>T\}}$ and $\mathbb{1}_{\{t<\tau \leq T\}}=\mathbb{1}_{\{\tau>t\}}-\mathbb{1}_{\{\tau>T\}}$. Following Filipovič (2009), we remark that every $\mathcal{F}_{\infty}^{W}$-measurable random variable satisfies $\mathbb{E}\left[X \mid \mathcal{F}_{t}\right]=$ $\mathbb{E}\left[X \mid \mathcal{F}_{t}^{W}\right]$ with $\tilde{\mathbb{P}}\left(\tau>t \mid \mathcal{F}_{\infty}^{W}\right)=\tilde{\mathbb{P}}\left(\tau>t \mid \mathcal{F}_{t}^{W}\right), t \geq 0$. Then,

$$
\begin{aligned}
\tilde{\mathbb{P}}(t<\tau & \left.\leq u \mid \mathcal{F}_{\infty}^{W} \vee \mathcal{F}_{t}^{N}\right)=\mathbb{1}_{\{\tau>t\}} e^{\int_{0}^{t} \tilde{h}(s) d s} \tilde{\mathbb{E}}\left[\mathbb{1}_{\{t<\tau \leq u\}} \mid \mathcal{F}_{\infty}^{W}\right] \\
& =\mathbb{1}_{\{\tau>t\}} e^{\int_{0}^{t} \tilde{h}(s) d s}\left(e^{-\int_{t}^{u} \tilde{h}(s) d s}-e^{-\int_{0}^{u} \tilde{h}(s) d s}\right) \\
& =\mathbb{1}_{\{\tau>t\}}\left(1-e^{-\int_{t}^{u} \tilde{h}(s) d s}\right) .
\end{aligned}
$$

This is the $\mathcal{F}_{\infty}^{W} \vee \mathcal{F}_{t}^{N}$ conditional distribution of $\tau \mid \tau>t$ and on differentiating (D.2) with respect to $u$ yields

$$
\mathbb{1}_{\{\tau>t\}} \tilde{h}(u) e^{-\int_{t}^{u} \tilde{h}(s) d s} \mathbb{1}_{\{\tau \leq u\}} .
$$

Then the expectation at the numerator can be simplified as follows

$$
\begin{gathered}
\tilde{\mathbb{E}}\left[e^{-\int_{t}^{\tau} r(s) d s} \mathbb{1}_{\{t<\tau \leq T\}} \mid \mathcal{F}_{t}\right]=\tilde{\mathbb{E}}\left[\tilde{\mathbb{E}}\left[e^{-\int_{t}^{\tau} r(s) d s} \mathbb{1}_{\{t<\tau \leq T\}} \mid \mathcal{F}_{\infty}^{W} \vee \mathcal{F}_{t}^{N}\right] \mid \mathcal{F}_{t}\right] \\
=\mathbb{1}_{\{\tau>t\}} \tilde{\mathbb{E}}\left[\int_{t}^{T} \tilde{h}(u) e^{-\int_{t}^{u} r(s) d s} e^{-\int_{t}^{u} \tilde{h}(s) d s} d u \mid \mathcal{F}_{t}\right] \\
=\mathbb{1}_{\{\tau>t\}} \int_{t}^{T} \tilde{\mathbb{E}}\left[\tilde{h}(u) e^{-\int_{t}^{u}(r(s)+\tilde{h}(s)) d s} \mid \mathcal{F}_{t}^{W}\right] d u
\end{gathered}
$$

In addition, the first expectation at the numerator can be simplified as

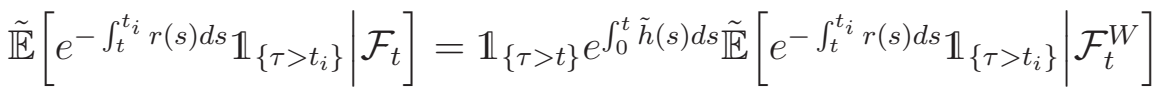

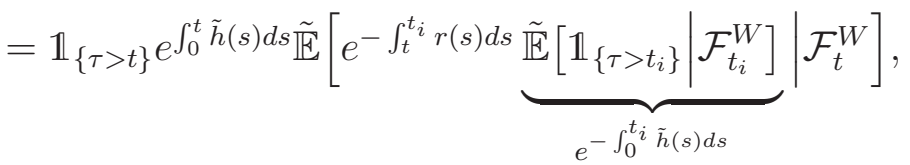

$$
\begin{aligned}
& =\mathbb{1}_{\{\tau>t\}} \tilde{\mathbb{E}}\left[e^{-\int_{t}^{t_{i}}(r(s)+\tilde{h}(s)) d s} \mid \mathcal{F}_{t}^{W}\right]=\mathbb{1}_{\{\tau>t\}} \bar{P}^{d}\left(t, t_{i}\right) .
\end{aligned}
$$

Substituting (D.4) and (D.3) into (D.1) we then have that

$$
\sum_{i=1}^{N} \tilde{\mathbb{E}}\left[e^{-\int_{t}^{t_{i}} r(s) d s} \mathbb{1}_{\left\{\tau>t_{i}\right\}} \mid \mathcal{F}_{t}\right]=\mathbb{1}_{\{\tau>t\}} \sum_{i=1}^{N} \bar{P}^{d}\left(t, t_{i}\right)
$$

and

$$
\tilde{\mathbb{E}}\left[e^{-\int_{t}^{\tau} r(s) d s} \mathbb{1}_{\{t<\tau \leq T\}} \mid \mathcal{F}_{t}\right]=\mathbb{1}_{\{\tau>t\}} \int_{t}^{T} \tilde{\mathbb{E}}\left[\tilde{h}(u) e^{-\int_{t}^{u}(r(s)+\tilde{h}(s)) d s} \mid \mathcal{F}_{t}^{W}\right] d u .
$$

from which (4.5) follows. 


\section{E Proof of Corollary 4.2 for Postponed CDS}

In the case of a postponed CDS, the value of premium leg will not have the accrual component and the CDS spread at time $t$ can be expressed as

$$
\tilde{\pi}(t)=\frac{(1-\mathcal{R}) \sum_{i=1}^{N} \tilde{\mathbb{E}}\left[e^{-\int_{t}^{t_{i}} r(s) d s} \mathbb{1}_{\left\{t_{i-1}<\tau \leq t_{i}\right\}} \mid \mathcal{F}_{t}\right]}{\sum_{i=1}^{N} \delta_{i} \tilde{\mathbb{E}}\left[e^{-\int_{t}^{t_{i}} r(s) d s} \mathbb{1}_{\left\{\tau>t_{i}\right\}} \mid \mathcal{F}_{t}\right]} .
$$

The conditional expectation in the numerator can then be simplified to

$$
\begin{aligned}
\tilde{\mathbb{E}}\left[e^{-\int_{t}^{t_{i}} r(s) d s}\right. & \left.\mathbb{1}_{\left\{t_{i-1}<\tau<t_{i}\right\}} \mid \mathcal{F}_{t}\right]=\tilde{\mathbb{E}}\left[\tilde{\mathbb{E}}\left[e^{-\int_{t}^{t_{i}} r(s) d s}\left(\mathbb{1}_{\left\{\tau<t_{i-1}\right\}}-\mathbb{1}_{\left\{\tau<t_{i}\right\}}\right) \mid \mathcal{F}_{t_{i}}^{W} \vee \mathcal{F}_{t}^{N}\right] \mid \mathcal{F}_{t}\right] \\
& \left.=\mathbb{1}_{\tau>t} \tilde{\mathbb{E}}\left[e^{-\int_{t}^{t_{i}} r(s) d s}\left(e^{-\int_{t}^{t_{i-1}} \tilde{h}(s) d s}-e^{-\int_{t}^{t_{i}} \tilde{h}(s) d s}\right)\right] \mid \mathcal{F}_{t}^{W}\right] \\
& =\mathbb{1}_{\tau>t}\left\{\tilde{\mathbb{E}}\left[e^{-\int_{t}^{t_{i}} r(s) d s} e^{-\int_{t}^{t_{i-1}} \tilde{h}(s) d s} \mid \mathcal{F}_{t}^{W}\right]-\tilde{\mathbb{E}}\left[e^{-\int_{t}^{t_{i}} r(s) d s} e^{-\int_{t}^{t_{i}} \tilde{h}(s) d s} \mid \mathcal{F}_{t}^{W}\right]\right\} \\
& =\mathbb{1}_{\tau>t}\left\{\tilde{\mathbb{E}}\left[e^{-\int_{t}^{t_{i}} r(s) d s} e^{-\int_{t}^{t_{i-1}} \tilde{h}(s) d s} \mid \mathcal{F}_{t}^{W}\right]-\bar{P}^{d}\left(t, t_{i}\right)\right\} .
\end{aligned}
$$

Following Brigo and Morini (2005), by using the approximation $e^{-\int_{t}^{t_{i}} r(s) d s} \approx e^{-\int_{t}^{t_{i-1}} r(s) d s}$, then we can write

$$
\left\{\begin{array}{l}
\tilde{\mathbb{E}}\left[e^{-\int_{t}^{t_{i}} r(s) d s} e^{-\int_{t}^{t_{i-1}} \tilde{h}(s) d s} \mid \mathcal{F}_{t}^{W}\right]=\bar{P}^{d}\left(t, t_{i-1}\right) \\
\tilde{\mathbb{E}}\left[e^{-\int_{t}^{t_{i}} r(s) d s} \mathbb{1}_{\left\{t_{i-1}<\tau<t_{i}\right\}} \mid \mathcal{F}_{t}\right]=\bar{P}^{d}\left(t, t_{i-1}\right)-\bar{P}^{d}\left(t, t_{i}\right) .
\end{array}\right.
$$

Hence the proof.

\section{References}

Amin, K. and Morton, R. (1995), 'Implied Volatility Functions in Heath-Jarrow-Morton Models', Journal of Financial Economics 35(2), 141-180.

Ball, C. A. and Torous, W. N. (1999), 'The Stochastic Volatility of Short-term Interest Rates: Some International Evidence', Journal of Finance 54(6), 2339-2359.

Bedendo, M., Cathcart, L. and El-Jahel, L. (2007), 'The Slope of the Term Structure of Credit Spreads: An Empirical Investigation', Journal of Financial Research 30(2), 237 - 257.

Berndt, A., Ritchken, P. and Sun, Z. (2010), 'On Correlation and Default Clustering in Credit Markets', Review of Financial Studies 23(7), 2680-2729.

Bielecki, T., Jeanblanc, M. and Rutkowski, M. (2007), 'Hedging of basket credit derivatives in credit swap market', Journal of Credit Risk 3(1), 91-132. 
Björk, T., Kabanov, Y. and Runggaldier, W. (1997), 'Bond Market Structure in the Presence of Marked Point Processes', Mathematical Finance 7(2), 211-239.

Björk, T., Landén, C. and Svensson, L. (2004), 'Finite-dimensional Markovian realizations for stochastic volatility forward-rate models', Proceedings of Royal Society London 460,(2041), 53-83.

Brigo, D. and Morini, M. (2005), 'CDS Market Formulas and Models', Invited Presentation at XVIII Warwick Option Conference .

Casassus, J., Collin-Dufresne, P. and Goldstein, R. (2005), 'Unspanned Stochastic Volatility and Fixed Income Derivatives Pricing', Journal of Banking and Finance 29, 2723-2749.

Chan, K. C., Karolyi, G. A., Longstaff, F. A. and Sanders, A. B. (1992), 'An Empirical Comparison of Alternative Models of the Short-Term Interest Rate', Journal of Finance 47, 1209-1227.

Chen, L. and Filipovič, D. (2007), 'Credit Derivatives in an Affine Framework', Asia-Pacific Financial Markets 14, 123-140.

Chiarella, C., Fanelli, V. and Musti, S. (2011), 'Modelling the Evolution of Credit Spreads Using the Cox Process within the HJM Framework: A CDS Option Pricing Model', European Journal of Operational Research .

Chiarella, C. and Kwon, O. (2000a), 'A Class of Heath-Jarrow-Morton Term Structure Models with Stochastic Volatility', QFRC Working Paper No. 34, School of Finance and Economics, University of Technology, Sydney .

Chiarella, C. and Kwon, O. (2000b), 'A Complete Markovian Stochastic Volatility Model in the HJM Framework', Asia Pacific Financial Markets 7(4), 293-304.

Chiarella, C. and Kwon, O. (2001), 'Classes of Interest Rate Models under the HJM Framework', Asia-Pacific Financial Markets 8(1), 1-22.

Chiarella, C. and Kwon, O. (2003), 'Finite Dimensional Affine Realisations of HJM Models in Terms of Forward Rates and Yields', Review of Derivatives Research 6(2), 129-155.

Chiarella, C., Maina, S. C. and Nikitopolous-Sklibosios, C. (2010), 'Markovian Defaultable HJM Term Structure Models with Unspanned Stochastic Volatility', QFRC Working Paper No. 283, School of Finance and Economics, University of Technology, Sydney .

Chiarella, C., Nikitopoulos-Sklibosios, C. and Schlögl, E. (2007), 'A Markovian Defaultable Term Structure model with State Dependent Volatilities', International Journal of Theoretical and Applied Finance 10(1).

Collin-Dufresne, P. and Goldstein, R. (2002), 'Do Bonds Span the Fixed Income Markets? Theory and Evidence for Unspanned Stochastic Volatility', Journal of Finance 57, 1685-1730.

D'Souza, D., Amir-Atefi, K. and Racheva-Jotova, B. (2004), Valuation of a Credit Spread Put Option: The Stable Paretian Model with Copulas, Birkhauser. Handbook of Computational and Numerical Methods in Finance.

Filipovič, D. (2009), Term Structure Models: A Graduate Course, Springer Finance. 
Heath, D., Jarrow, R. A. and Morton, A. (1992), 'Bond Pricing and the Term Structure of Interest Rates: A New Methodology for Contingent Claims Valuation', Econometrica 60, 77-105.

Heston, S. (1993), 'A Closed-Form Solution for Options with Stochastic Volatility with Applications to Bond and Currency Options', Review of Financial Studies 6, 327-343.

Hull, J. C. and White, A. (2001), 'Valuing Credit Default Swaps II: Modelling Default Correlation', Journal of Derivatives 8, 12-22.

Jabbour, G. M., El-masri, F. and Young, S. D. (2008), 'On the Lognormality of Forward Credit Default Swap Spreads', Journal of Financial Transformation 22, 41-48.

Jarrow, R. A. and Yu, F. (2001), 'Counterparty Risk and the Pricing of Defaultable Securities', The Journal of Finance 56, 1765-1799.

Krekel, M. and Wenzel, J. (2006), 'A Unified Approach to Credit Default Swaption and Constnat Default Swap Valuation', Berichte des Fraunhofer ITWM 96.

Lando, D. (1998), 'On Cox Processes and Credit Risky Securities', Review of Derivatives Research $\mathbf{2}(2 / 3), 99-120$.

Li, H. and Zhao, F. (2006), 'Unspanned Stochastic Volatility: Evidence from Hedging Interest Rate Derivatives', Journal of Finance 61, 341-378.

Maina, S. C. (2011), 'Credit Risk Modelling in Markovian HJM Term Structure Class of Models with Stochastic Volatility', Ph.D Thesis - School of Finance and Economics, University of Technology, Sydney .

Mercurio, F. and Moraleda, J. M. (2000), 'An Analytically Tractable Interest Rate Model with Humped Volatility', European Journal of Operational Research 120, 205-214.

Pan, J. and Singleton, K. K. (2008), 'Default and Recovery Implicit in the Term Structure of Sovereign CDS Spreads', Journal of Finance 63(5), 2345-2384.

Reno, R. and Uboldi, A. (2005), 'On the Presence of Unspanned Volatility in European Interest Rate Options', Applied Financial Economics Letters 1, 15-18.

Rutkowski, M. and Armstrong, A. (2009), 'Valuation of Credit Default Swaptions and Credit Default Index Swaptions', International Journal of Theoretical and Applied Finance 12(7), 1027-1053.

Schönbucher, P. (1998), 'Term Structure Modelling of Defaultable Bonds', Review of Derivatives Research 2, 161-192.

Schönbucher, P. (2003), Credit Derivatives Pricing Models: Model, Pricing and Implementation, John Wiley \& Sons.

Schönbucher, P. (2004), 'A Measure of Survival', Risk January.

Schönbucher, P. J. and Schubert, D. (2001), 'Copula-Dependent Default Risk in Intensity Models', University of Bonn. Working Paper, December.

Trolle, A. B. and Schwartz, E. S. (2009), 'A General Stochastic Volatility Model for the Pricing of Interest Rate Derivatives', The Review of Financial Studies 22(5), 2007-2057. 